\title{
Influence of prefoldin subunit 4 on the tolerance of Kluyveromyces marxianus to lignocellulosic biomass-derived inhibitors
}

Nini Zhang ${ }^{1 \dagger}$, Yingying Shang ${ }^{1 \dagger}$, Feier Wang ${ }^{1}$, Dongmei Wang ${ }^{1,3^{*}}$ and Jiong Hong ${ }^{1,2,3^{*}}$ (D)

\begin{abstract}
Background: Kluyveromyces marxianus is a potentially excellent host for microbial cell factories using lignocellulosic biomass, due to its thermotolerance, high growth rate, and wide substrate spectrum. However, its tolerance to inhibitors derived from lignocellulosic biomass pretreatment needs to be improved. The prefoldin complex assists the folding of cytoskeleton which relates to the stress tolerance, moreover, several subunits of prefoldin have been verified to be involved in gene expression regulation. With the presence of inhibitors, the expression of a gene coding the subunit 4 of prefoldin (KmPFD4), a possible transcription factor, was significantly changed. Therefore, KmPFD4 was selected to evaluate its functions in inhibitors tolerance.

Results: In this study, the disruption of the prefoldin subunit 4 gene (KmPFD4) led to increased concentration of intracellular reactive oxygen species (ROS) and disturbed the assembly of actin and tubulin in the presence of inhibitors, resulting in reduced inhibitor tolerance. Nuclear localization of KmPFD4 indicated that it could regulate gene expression. Transcriptomic analysis showed that upregulated gene expression related to ROS elimination, ATP production, and NAD ${ }^{+}$synthesis, which is a response to the presence of inhibitors, disappeared in KmPFD4-disrupted cells. Thus, KmPFD4 impacts inhibitor tolerance by maintaining integration of the cytoskeleton and directly or indirectly affecting the expression of genes in response to inhibitors. Finally, overexpression of KmPFD4 enhanced ethanol fermentation with a $46.27 \%$ improvement in productivity in presence of the inhibitors.
\end{abstract}

Conclusion: This study demonstrated that KmPFD4 plays a positive role in the inhibitor tolerance and can be applied for the development of inhibitor-tolerant platform strains.

Keywords: Prefoldin, KmPFD4, Lignocellulosic biomass, Inhibitor tolerance, Kluyveromyces marxianus

\section{Background}

Global energy consumption could reach more than $10^{6}$ quadrillion British thermal unit by 2050 [1]. With increased demand for energy and chemicals, more fossil fuels are being consumed. Not only have the limited crude oil and coal supplies become depleted, but

\footnotetext{
*Correspondence: dmwang09@ustc.edu.cn; hjiong@ustc.edu.cn ${ }^{\dagger}$ Zhang Nini and Shang Yingying contributed equally to this work 1 School of Life Sciences, University of Science and Technology of China, Hefei 230027, Anhui, People's Republic of China

Full list of author information is available at the end of the article
}

excessive consumption of fossil fuels has led to global warming due to the emission of large amounts of greenhouse gases and organic pollutants. Therefore, more attention is being paid to the development of biofuel and green chemicals. Lignocellulosic biomass is an important feedstock for bioethanol and other chemicals because it is renewable and sustainable. In general, more than 220 billion tons of lignocellulosic biomass is produced annually [2]. Large amounts of lignocellulosic biomass make it possible, at least partly, to substitute fossil fuel energy and resources. Lignocellulosic biomasses are more original author(s) and the source, provide a link to the Creative Commons licence, and indicate if changes were made. The images or other third party material in this article are included in the article's Creative Commons licence, unless indicated otherwise in a credit line to the material. If material is not included in the article's Creative Commons licence and your intended use is not permitted by statutory regulation or exceeds the permitted use, you will need to obtain permission directly from the copyright holder. To view a copy of this licence, visit http://creativecommons.org/licenses/by/4.0/. The Creative Commons Public Domain Dedication waiver (http://creativeco mmons.org/publicdomain/zero/1.0/) applies to the data made available in this article, unless otherwise stated in a credit line to the data. 
attractive than corn and sugarcane because they do not compete with food [3].

Pretreatment is required to improve the efficiency of lignocellulosic biomass hydrolysis and the fermentable sugar yield. Lignocellulosic biomass is mainly composed of cellulose, hemicellulose, and lignin with a ratio in the range of 30-55 wt $\%, 15-40 \mathrm{wt} \%$, and $10-35 \mathrm{wt} \%$, respectively [1]. Glucose, xylose, arabinose, and other sugars can be released from lignocellulosic biomass through enzymatic hydrolysis [4]. However, crystallized cellulose, hemicellulose, and highly polymerized phenolic lignin lead to difficulty in lignocellulosic biomass hydrolysis [5]. Therefore, pretreatment, which can destroy the structure of lignocellulosic biomasses, improves accessibility and enables easy hydrolysis. During pretreatment, inhibitors which inhibit the growth and the fermentation ability of microbes are produced. These inhibitors are mainly weak acids, furan compounds, and phenolic chemicals. Weak acids include formic acid, acetic acid, and levulinic acid. Furfuran compounds generally refer to furfural and 5-hydroxymethyl-2-furaldehyde (HMF), whereas phenolic chemicals are mainly the compounds from the degradation of lignin and other aromatic compounds obtained from biomass $[4,6]$.

Furfural and 5-hydroxymethylfurfural (HMF) can negatively affect the microbial fermentation by inhibiting cell growth and sugar uptake rate, subsequently reducing ethanol production rate. Furfuran compounds also have negative effects on metabolisms, cell wall formation, and DNA, RNA and/or protein synthesis [7]. The primary carbon catabolism enzymes including acetaldehyde dehydrogenase, alcohol dehydrogenase, aldehyde dehydrogenase, glyceraldehydre-3-phosphate dehydrogenase, and pyruvate dehydrogenase are also repressed by furfuran compounds [6-9]. Inhibition mechanisms of phenolic compounds on eukaryotic microorganisms have not yet been completely elucidated [8]. Phenolic compounds lead to a loss of integrity in cell membrane membranes, thereby affecting their ability to serve as selective barriers and enzyme matrices. Consequently, phenolic compounds reduce cell growth, sugar assimilation, and fermentation [6]. Phenolic compounds are also able to penetrate cell membranes and damage internal structures, as well as causing changes in the morphology of cells [6]. Weak acids reduce the cytosolic pH [10]. The protons, then, are pumped out of the cell through the plasma membrane ATPase with ATP hydrolysis. Consequently, less ATP is available for biomass formation. With the presence of higher concentrations, the ATP requirements increased and cells cytosol is acidified [11]. Furfuran compounds, acetic acid, and phenolic compounds lead to accumulation of reactive oxygen species (ROS) in yeast, and subsequently cause cellular damage including damage to mitochondria and vacuole membranes, the actin cytoskeleton and nuclear chromatin [12,13].

Yeast cells metabolize or pump out the inhibitors to tolerate the inhibitors. Phenolic compounds in the inhibitors can be converted to less toxic compounds. For instance, coniferyl aldehyde is reduced to coniferyl alcohol and dihydroconiferyl alcohol. Furfurals are metabolized into less toxic acid or alcohol forms using NAD $(\mathrm{P}) \mathrm{H}$ as cofactor [11]. The furfurals and phenolic compounds were also proved to be reduced to corresponding alcohol in K. marxianus by Oliva group and in our previous study $[14,15]$. However, when the concentration of inhibitors is high, the $\mathrm{NAD}(\mathrm{P}) \mathrm{H}$ and ATP for the inhibitors conversion, ROS elimination and inhibitors pumping out are not enough, the growth of the yeast is hindered, even death under severe conditions.

Improvement of inhibitor tolerance of microbes could enhance fermentation and reduce the cost of inhibitors removal. The lignocellulosic biomass-derived inhibitors can be removed through physical, chemical, or biological methods [16]. However, inhibitors removal by physical or chemical methods incurs greater costs in industrial production. Many microorganisms can degrade the lignocellulosic biomass-derived inhibitors, although this procedure is generally time-consuming. The construction of genetically engineered microbes with improved inhibitor tolerance is another important approach that can prevent or reduce the cost of inhibitors removal [8].

Studies regarding the inhibitor tolerance mechanism of Kluyveromyces marxianus are necessary and can improve its inhibitor tolerance. K. marxianus is a nontraditional yeast and generally regarded as safe [17]. Its thermotolerance enables fermentation at elevated temperatures, which can be used in the tropic region and high temperature season with reduced contamination [18-21]. K. marxianus is considered to be the fastestgrowing eukaryote with highest possible replication rate [22] and the high growth rate of $K$. marxianus could improve the production rate. The pentose utilization ability of $K$. marxianus is attractive for fermentation with lignocellulosic biomass hydrolysate. The combination of thermotolerance and wide substrate spectrum makes $K$. marxianus suitable for the simultaneous saccharification and fermentation or simultaneous saccharification and co-fermentation of lignocellulosic biomass [15, 23-25]. K. marxianus has many advantages, and its tolerance to single kind inhibitor is higher than that of the widely used Saccharomyces cerevisiae [14, 26-28]. However, it is still necessary to improve the tolerance of $K$. marxianus to multiple inhibitors.

Prefoldin is a co-chaperone that is widely present in archaea and eukaryotes, and facilitates the supply of unfolded or partially folded substrates to class II 
chaperonin chaperonin-containing TCP-1. In canonical prefoldin, four $\beta$-type subunits (eukaryotic PFD1, 2, 4 , and 6) form two dimers onto two subunits of $\alpha$ type (PFD3 and 5) [29]. The canonical prefoldin complex helps actin and tubulin assembly and the folding of other proteins with chaperones [30]. Prefoldin is also a gene regulator [29]. The increased levels of subunit PFD1 represses cyclin A expression by directly interacting with its promoter at the transcriptional start site [31]. PFD3 influences the action of the viral $\mathrm{HBx}$ protein as a transcriptional coactivator [32]. PFD4 is reported with possible transcription factor activity in human cells [33]. The subunit PFD5 acts as a co-repressor of the E-box-dependent transactivation activity of c-Myc [34]. Subunits PFD5 and PFD6 play a relevant role in gene expression in relation to DELLA nuclear factors, which are known to regulate the expression of a large set of genes in plants[35]. Finally, yeast prefoldin subunits PFD1, PFD2, PFD5 and PFD6 bind yeast chromatin in a transcription-dependent manner following a profile that parallels the phosphorylation of the Ser2 residues of RNA pol II C-terminal domain, and play a positive role in transcription elongation [36]. However, the functions of prefoldin in the tolerance to lignocellulosic biomass-derived inhibitors have not been reported.

Our transcriptomic analysis illustrated that, with the presence of multiple lignocellulosic biomass-derived inhibitors, the subunits of prefoldin in $K$. marxianus except PFD5 are down-regulated (Additional file 1). The real-time PCR (RT-PCR) results indicated that compared to the expression at exponential phase, the expression of most prefoldin subunits increased at stationary phase except the KmPFD1expression with no inhibitor and KmPFD4 expression with inhibitors (Additional file 2: Fig. S1). Since PFD4 has not been as well studied as other subunits, KmPFD4 in K. marxianus was disrupted or overexpressed to evaluate its effect on the tolerance to inhibitors. The effect of the disruption of KmPFD4 on actin and tubulin, the intracellular location of KmPFD4, intracellular ROS, and the transcriptome were determined to elucidate the mechanism of inhibitor tolerance. Furthermore, the effect of disruption on the tolerance towards other kinds of stress, such as salt tolerance, temperature, etc., was evaluated as well. Finally, the effect of overexpressing KmPFD4 in ethanol fermentation in the presence of inhibitors was also evaluated.

\section{Materials and methods}

\section{Reagents and original strain}

D-glucose, D-xylose, and yeast nitrogen base without amino acids (YNB) were obtained from Sangon Biotech Co. (Shanghai, China). Restriction endonucleases were purchased from Thermo Fisher Scientific (West
Palm Beach, Florida, USA). Yeast extract, tryptone, and bacteriological peptone were acquired from Oxoid (Oxoid Ltd., Basingstoke, Hampshire, UK). K. marxianus YHJ010, a trp1, leu2, and ura3 auxotroph derived from K. marxianus NBRC 1777, was used as the original strain [37]. Synthetic dropout (SD) medium (6.7 g/L YNB, $20 \mathrm{~g} / \mathrm{L}$ glucose) was used for transformant selection. Yeast extract/peptone-dextrose (YPD) medium (10 g/L yeast extract, $20 \mathrm{~g} / \mathrm{L}$ bacteriological peptone, and $20 \mathrm{~g} / \mathrm{L}$ D-glucose) was used for the cultivation of $K$. marxianus strains. To prepare solid plates of each medium, $1.5 \%$ (w/v) agar was added. Escherichia coli DH5 $\alpha$ was used as the host for gene cloning and vector construction, and was cultivated in lysogeny broth medium supplemented with $100 \mu \mathrm{g} / \mathrm{mL}$ ampicillin.

\section{Plasmids construction}

DNA fragment of prefoldin subunit 4 (KmPFD4, GenBank accession No. BAP73153, locus_tag: KMAR_60414) was amplified from the genomic DNA of $K$. marxianus YHJ010 with primers KmPFD4-F and KmPFD4-R (Additional file 2: Table S1). The amplified fragment was inserted into pGEM-T easy (Promega Corporation, Madison, WI, USA), and the resultant plasmid was pSY001 (Table 1).

The KmPFD4 disruption cassette was constructed in plasmid pSY002. The frame of plasmid pSY001 and part of KmPFD4 were amplified with primers KmPFD4-F2 and KmPFD4-R2 (Additional file 2: Table S1; Fig. 1). After the ScURA3 expression cassette including the original promoter, ORF, and terminator of ScURA3 was amplified from YEUGAP [17] with primers ScURA3-SmaI-F and ScURA3-SmaI-R (Additional file 2: Table S1), it was ligated with the amplified pSY001 frame (Fig. 1). The obtained plasmid was pSY002 (Table 1). The KmPDF4 disruption cassette in pSY002 included 644 bp upstream and 621 bp downstream homologous recombination sequences of KmPDF4 and ScURA3 expression cassette (Fig. 1).

The KmPFD4 overexpression plasmid was constructed as follows: the open reading frame (ORF) of KmPFD4 was amplified from pYS001 with primers KmPFD4-EcoRIF and KmPFD4-NotI-R (Additional file 2: Table S1) and inserted into the YEGAP plasmid [17] at EcoRI and NotI sites. The resultant plasmid was named pSY003 (Table 1).

Two plasmids were constructed for the intracellular localization of KmPFD4. The enhanced green florescent protein (EGFP) gene was amplified from pPCG [39] with the primers EGFP-EcoRI-F and EGFP-NotI-R and inserted into YEGAP between EcoRI and NotI sites. The resultant plasmid pSY004 (Table 1) was used to construct a control strain for EGFP expression in K. marxianus. The ORF of KmPFD4 was amplified from pSY001 
Table 1 Plasmids used in this study

\begin{tabular}{|c|c|c|}
\hline Plasmids & Description & References \\
\hline YEGAP & $A m p^{R}, S_{C} T R P 1, P_{S C G A P D H} T_{S C G A P D H}$ & [37] \\
\hline YEUGAP & $A m p^{R}, S C \cup R A 3, P_{S C G A P D H}, T_{S C G A P D H}$ & [17] \\
\hline YEUKmPGK & $A m p^{R}, S_{C} \cup R A 3, P_{K m P G K_{1}} T_{S C G A P D H}$ & [38] \\
\hline pGEM-T Easy & $A m p^{R}$ & Promega \\
\hline pGEM-T- $\triangle S C U R A 3$ & $A m p^{R}$, nonfunctional ScURA3 & [18] \\
\hline PPCG & Zeocin $^{R}$, CBM-EGFP- pPICZ aA & [39] \\
\hline pSY001 & $\begin{array}{l}\text { Amp } p^{R}, K m P F D 4-p G E M-T \text { Easy vector } \\
\text { AmpR, KmPFD4-T vector }\end{array}$ & This study \\
\hline pSY002 & $A m p^{R}, K m P F D 4$ inserted with ScURA3 & This study \\
\hline pSY003 & $A m p^{R}, S_{C T R P 1} P_{S C G A P D H^{-}} K m P F D 4-T_{S C G A P D H}$ & This study \\
\hline pSY004 & $A m P^{R}, S C U R A 3, P_{S C G A P D H}-E G F P-T_{S C G A P D H}$ & This study \\
\hline pSY005 & $A m P^{R}, S c \cup R A 3, P_{S C G A P D H^{-}} K m P F D 4-E G F P-T_{S \subset G A P D H}$ & This study \\
\hline
\end{tabular}

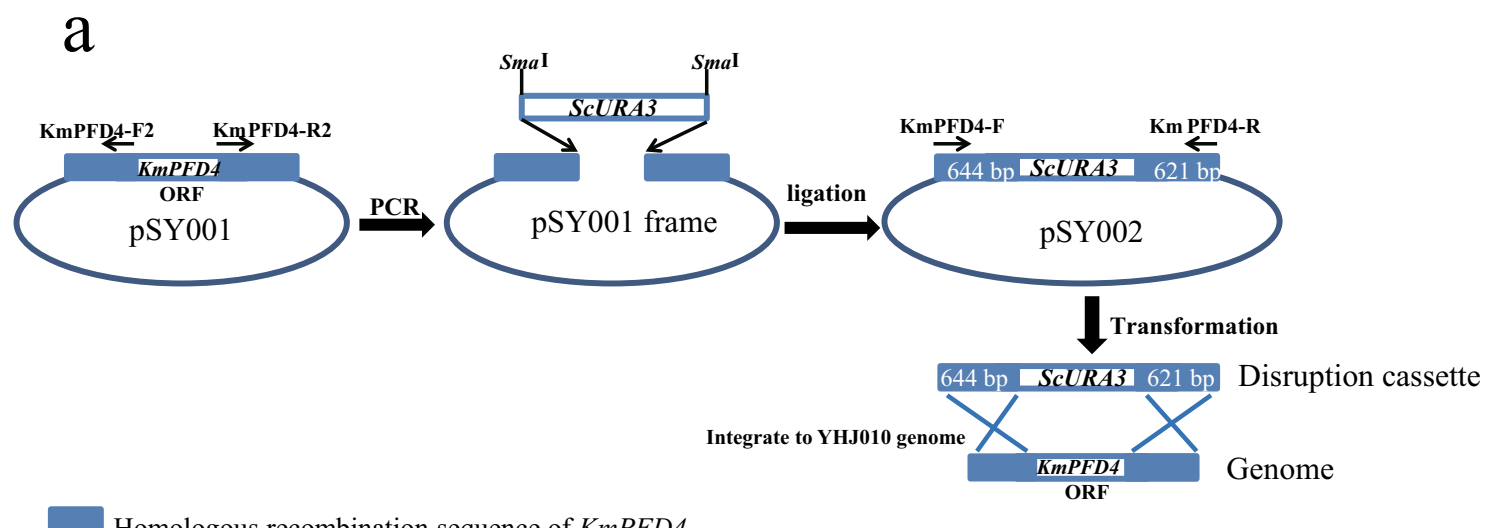

Homologous recombination sequence of KmPFD4

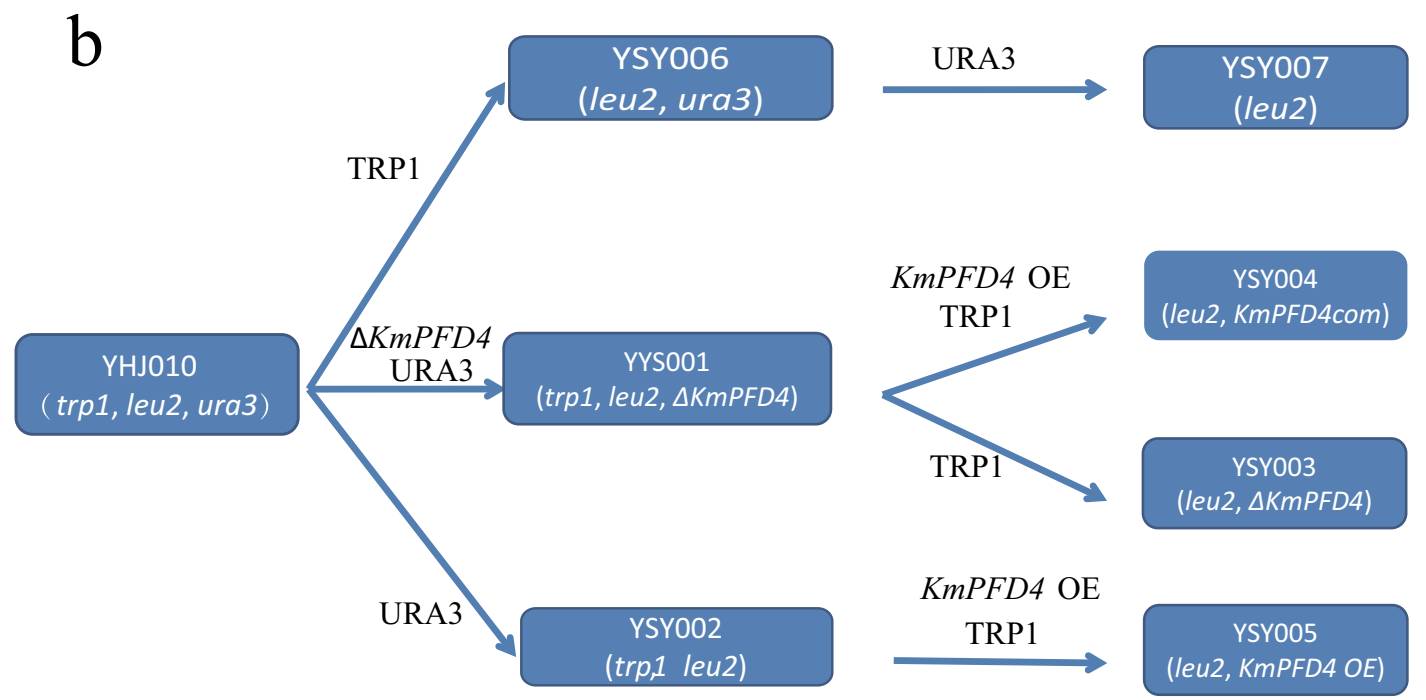

Fig. 1 Schematic diagram for the construction of strains. a Construction of the KmPFD4 gene disruption cassette and gene disruption, $\mathbf{b}$ The strain construction procedure. $\triangle$ : disruption, OE: overexpression 
with primers KmPFD4-EcoRI-F and KmPFD4-fusion-R. At the same time, the EGFP gene was amplified from pPCG with the primers EGFP-fusion-F and EGFP-NotI$\mathrm{R}$. The resultant KmPFD4 and EGFP DNA fragments were fused together with primers KmPFD4-EcoRI-F and EGFP-NotI-R through overlap extension PCR. The fused KmPFD4-EGFP fragment was digested with EcoRI and Not I and inserted into YEGAP. The resultant plasmid was pSY005 (Table 1).

\section{Strains construction}

The KmPFD4 disrupted strain was constructed with YHJ010. The gene disruption cassette was amplified from pSY002 using primers KmPFD4-F and KmPFD4-R and transformed into YHJ010 by the lithium acetate method [40]. After screening on SD medium supplemented with leucine and tryptophan, the positive clones were confirmed by PCR with genomic DNA as the template. The obtained KmPFD4-disrupted strain was named YSY001 (Table 2). Subsequently, the empty plasmids YEGAP or pSY003 were transformed into YSY001, and the obtained strains were YSY003 and YSY004, respectively (Table 2). YSY003 was a KmPFD4-disrupted URA3 and TRP1 auxotroph-complemented strain. YSY004 was a retrocomplementary strain of KmPFD4 disruption (Table 2). To confirm if more KmPFD4 could enhance the inhibitor tolerance of $K$. marxianus, the KmPFD4 was overexpressed with strong promoter (TDH3).

The ScURA3 expression cassette was amplified from YEUGAP with primers ScURA3-SmaI-F and ScURA3SmaI-R and transformed into YHJ010. The resultant strain was named YSY002, which is a wild-type KmPFD4 and selection marker-complemented strain. YSY002 was then transformed with the pSY003 and the resultant YSY005 was the KmPFD4 overexpression strain.

YHJ010 was transformed with the plasmid YEGAP [37] and ScURA3 expression cassette in turn to obtain

Table 2 Strains used in this study

\begin{tabular}{|c|c|c|}
\hline Strains & Description & References \\
\hline YHJ010 & $\begin{array}{l}\text { K. marxianus NBRC1777, } \triangle K m U R A 3:: \text { Kanr, } \\
\triangle K m L E U 2:: \text { hisG, } \triangle K m T R P 1:: \text { hisG }\end{array}$ & [37] \\
\hline YSY001 & K. marxianus, YHJ010, $\triangle$ KmPFD4::SCURA3 & This study \\
\hline YSY002 & K. marxianus, YHJ010, ScURA3 & This study \\
\hline YSY003 & K. marxianus YSY001, SCTRP1 & This study \\
\hline YSY004 & K. marxianus YSY001, ScTRP1, KmPFD4 & This study \\
\hline YSY005 & K. marxianus YSY002, ScTRP1, KmPFD4 & This study \\
\hline YSY006 & K. marxianus YHJ010, ScTRP1 & This study \\
\hline YSY007 & K. marxianus YSY006, ScURA3 & This study \\
\hline YSY008 & K. marxianus YHJ010, EGFP & This study \\
\hline YSY009 & K. marxianus YHJ010, KmPFD4-EGFP & This study \\
\hline
\end{tabular}

YSY006 and YSY007.YSY007 was the URA3 and TRP1 auxotroph marker-complemented strain, which was used as a wild-type control.

pSY004 or pSY005 was linearized and transformed into YHJ010. YSY008 and YSY009 were obtained and used for intracellular localization of KmPFD4. These strains expressed EGFP and KmPFD4-EGFP, respectively.

\section{Growth assay of KmPFD4 disruption or overexpression on inhibitors or other stress tolerance}

YSY003, YSY004, YSY005, and YSY007 were inoculated into $5 \mathrm{~mL}$ of YPD and cultivated overnight at $42{ }^{\circ} \mathrm{C}$ with $250 \mathrm{rpm}$ shaking. The overnight cultures were inoculated into $30 \mathrm{~mL}$ of YPD medium (pH 6.0) containing no inhibitor, various inhibitors, $20 \mathrm{~g} / \mathrm{L}$ ethanol, $180 \mathrm{~g} / \mathrm{L}$ glucose, or $0.5 \mathrm{M} \mathrm{NaCl}$ with a starting $\mathrm{OD}_{600}=0.5$ in a $250 \mathrm{~mL}$ flask. Then, they were cultivated at $42{ }^{\circ} \mathrm{C}$ with shaking at $250 \mathrm{rpm}$. The $\mathrm{OD}_{600}$ was monitored during growth. Cultivation at $45^{\circ} \mathrm{C}$ was used as temperature stress condition. The inhibitors used included mixed inhibitors, $20 \mathrm{~g} / \mathrm{L}$ acetate sodium, $2.0 \mathrm{~g} / \mathrm{L}$ furfurals $(1.0 \mathrm{~g} / \mathrm{L}$ furfural and $1.0 \mathrm{~g} / \mathrm{L} \mathrm{HMF}$ ), or $1.3 \mathrm{~g} / \mathrm{L}$ phenols (4-hydroxybenzaldehyde, syringaldehyde, catechol, and vanillin with $0.325 \mathrm{~g} / \mathrm{L}$ of each compound). The mixed inhibitors contained $2.0 \mathrm{~g} / \mathrm{L}$ acetate sodium, $0.5 \mathrm{~g} / \mathrm{L}$ furfural, $0.5 \mathrm{~g} / \mathrm{L}$ HMF, $0.05 \mathrm{~g} / \mathrm{L}$ 4-hydroxybenzaldehyde, $0.05 \mathrm{~g} / \mathrm{L}$ syringaldehyde, $0.05 \mathrm{~g} / \mathrm{L}$ catechol, and $0.05 \mathrm{~g} / \mathrm{L}$ vanillin.

\section{Intracellular ROS assay}

The intracellular concentration of ROS was determined by 2,7-dichlorodihydrofluorescein diacetate (DCFH-DA) staining. Strains YSY003, YSY004, YSY005, and YSY007 were inoculated into $30 \mathrm{~mL}$ of YPD medium with starting $\mathrm{OD}_{600}=0.5$ and cultivated at $42{ }^{\circ} \mathrm{C}$ with $250 \mathrm{rpm}$ shaking. When the cell density reached $6\left(\mathrm{OD}_{600}\right)$, the cells were recovered by centrifugation at $5000 \times g$ and resuspended in YPD ( $\mathrm{pH}$ 6.0) containing mixed inhibitors and continuously cultured for $2 \mathrm{~h}$ at $42{ }^{\circ} \mathrm{C}$ with $250 \mathrm{rpm}$ shaking. The mixed inhibitors contained $5.3 \mathrm{~g} / \mathrm{L}$ acetate sodium, $1.3 \mathrm{~g} / \mathrm{L}$ furfural, $1.3 \mathrm{~g} / \mathrm{L}$ HMF, $0.125 \mathrm{~g} / \mathrm{L}$ 4-hydroxybenzaldehyde, $0.125 \mathrm{~g} / \mathrm{L}$ syringaldehyde, $0.125 \mathrm{~g} / \mathrm{L}$ catechol, and $0.125 \mathrm{~g} / \mathrm{L}$ vanillin. The cells were then recovered and stained with DCFH-DA [41]. Fluorescence was detected by using a CLARIOstar multimode microplate reader. The excitation and emission wavelengths were 488 and $525 \mathrm{~nm}$, respectively. The concentrations of inhibitors used in ROS determination were higher than those used in growth curve determination.

\section{Determine the effect of KmPFD4 to the respiratory efficiency of $K$. marxianus}

YSY003 or YSY007 strain was inoculated in $5 \mathrm{~mL}$ YPD medium and cultivated at $37{ }^{\circ} \mathrm{C}$ overnight. Then, the 
preculture was diluted 5000-fold with water, and $5 \mu \mathrm{l}$ cell suspension was inoculated on YPD plate which contained no or mixed inhibitors. The concentration of inhibitors was same as used in growth assay. After cultivation at $37^{\circ} \mathrm{C}$ for $18 \mathrm{~h}$, a top agar containing $1 \%(\mathrm{w} / \mathrm{v})$ agar, $0.5 \%(\mathrm{w} / \mathrm{v})$ glucose, and $0.005 \%(\mathrm{w} / \mathrm{v}) 2,3,5$-Triphenyltetrazolium chloride (TTC) was overlaid on the plate. Then the plate was incubated at $30^{\circ} \mathrm{C}$ for $3 \mathrm{~h}$. The lighter color of the colonies indicated respiratory deficiency [42].

\section{RNA-seq analysis of the KmPFD4-disrupted strain}

After the cells of $K$. marxianus YSY003 or YSY007 were cultivated with YPD (pH 6.0) containing mixed inhibitors and continuously cultured for $2 \mathrm{~h}$ at $42{ }^{\circ} \mathrm{C}$ with $250 \mathrm{rpm}$ shaking, the cells were then frozen in liquid nitrogen. The inhibitors concentration and culture conditions were the same as described in "Intracellular ROS assay" section. The cells cultivate in the YPD medium (pH6.0) without inhibitors were also collected as control. Total RNA extraction, cDNA library preparation, and RNA-seq analysis performed by Majorbio Co., Ltd (Shanghai, China). The clean reads were mapped to the reference genome of K. marxianus NBRC1777 from GenBank (accession No. AP014599-AP014607 using TopHat (http://ccb.jhu.edu/ software/tophat/index.shtml).

For gene function annotations, obtained unigene sequences were annotated by searching in various protein databases, including the National Center for Biotechnology Information (NCBI) non-redundant protein (NR) database, the NCBI NR nucleotide sequence (Nt) database, Swiss-Prot, Pfam, Cluster of Orthologous Groups of proteins (COG), Gene Ontology (GO), and Kyoto Encyclopedia of Genes and Genomes (KEGG).

For different gene expression analysis, transcripts per million (TPM) were used as a value of normalized gene expression, and genes were considered differentially expressed in a given library when $p<0.001$ and a greater than two-fold-change (FC) in expression across libraries was observed using the webtool DEGSeq (https://bioco nductor.org/packages/stats/bioc/DEGSeq/). These genes were annotated as differentially expressed genes (DEGs).

\section{Real-time PCR analysis}

Real-time PCR (RT-PCR) was used to analyze the expression level of each subunit of prefoldin and validation of RNA-seq results. Total RNA was isolated using a yeast total RNA extraction kit (Sangon Biotech Co. Shanghai, China). The genomic DNA in isolated RNA was removed by gDNA Eraser (SparkJade Science Co., Ltd., Qingdao, China) and cDNA was synthesized using the SPARKscript II RT Plus Kit (SparkJade Science Co., Ltd., Qingdao, China) [27]. Real-time PCR was conducted on a Roche LightCycler ${ }^{\circledR} 96$ (Roche Molecular Systems, Inc.,
CA, USA) using the ChamQ Universal SYBR qPCR Master Mix kit (Vazyme Biotech Co.,Ltd, Nanjing, China). The primers for each gene and the ACT1 internal control are shown in Additional file 2: Table S1. The cycle threshold values $\left(C_{\mathrm{T}}\right)$ were determined and the relative fold differences were calculated using the $2^{-\Delta \Delta C T}$ method [43] with $A C T 1$ as the endogenous reference gene.

\section{Evaluation of tolerance to inhibitors and stress by solid medium}

The cells of the evaluated strains were cultivated in YPD medium with a starting $\mathrm{OD}_{600}=0.5$ at $42{ }^{\circ} \mathrm{C}$ with $250 \mathrm{rpm}$ shaking until $\mathrm{OD}_{600}=6$. The cells were recovered by centrifugation at $12,000 \times \mathrm{g}$ and resuspended in sterilized water. Then, cells were diluted to $\mathrm{OD}_{600}=6 \times 10^{-1}, 6 \times 10^{-2}, 6 \times 10^{-3}$, and $6 \times 10^{-4}$ and $3 \mu \mathrm{L}$ of cells were spotted on the plates. For the inhibitor tolerance, the mixed inhibitors, including $2.0 \mathrm{~g} / \mathrm{L}$ acetic acid, $0.5 \mathrm{~g} / \mathrm{L}$ furfural, $0.5 \mathrm{~g} / \mathrm{L} \mathrm{HMF}$ and $0.2 \mathrm{~g} / \mathrm{L}$ phenols,were added to the YPD ( $\mathrm{pH}$ 6.0) solid medium. As to the single type of inhibitor treatment, $20 \mathrm{~g} / \mathrm{L}$ acetic sodium, the furfurals including $0.5 \mathrm{~g} / \mathrm{L}$ furfural and $0.5 \mathrm{~g} / \mathrm{L}$ HMF, or the phenols containing $1.3 \mathrm{~g} / \mathrm{L}$ phenols was added to the YPD ( $\mathrm{pH}$ 6.0) solid medium. To evaluate the effect on tubulin in the KmPFD4-disrupted strain, $7 \mu \mathrm{g} / \mathrm{mL}$ benomyl or $0.7 \mathrm{M} \mathrm{NaCl}$ was added to the YPD solid medium.

\section{Intracellular localization of KmPFD4}

To analyze the mechanism of KmPFD4 in inhibitor tolerance, intracellular localization was determined. YSY008 (EGFP) and YSY009 (KmPFD4-EGFP) were cultivated in YPD or YPD containing mixed inhibitors. YSY008 or YSY009 was cultivated at $42{ }^{\circ} \mathrm{C}$ until the $\mathrm{OD}_{600}$ reached 6. Then, $500 \mu \mathrm{L}$ of the cells were recovered by centrifugation at $12,000 \times g$ and stained with 4,6-diamidino-2-phenylindole (DAPI; Sigma, USA) as previously described (Amberg et al. [44]. The imaging data were collected using a Perkin Elmer Ultraview VoX Spinning Disk Microscope equipped with a Hamamatsu C910023B EMCCD camera and a CFI Apochromat TIRF $100 \times$ objective $(\mathrm{NA}=1.49)$. The excitation wavelengths for EGFP and DAPI were 488 and $405 \mathrm{~nm}$, respectively.

\section{Detecting the effect of KmPFD4 disruption on actin assembly}

The effect of KmPFD4 disruption on actin was detected using the TRITC phalloidin staining. YSY003 (KmPFD disrupted) and YSY007 (control) were cultivated in YPD medium at $42{ }^{\circ} \mathrm{C}$ with $250 \mathrm{rpm}$ shaking until $\mathrm{OD}_{600}=6$. Then, the cells were recovered and stained with $200 \mathrm{nM}$ TRITC phalloidin as previously described [45]. The imaging data were collected using a Perkin Elmer Ultraview 
VoX Spinning Disk Microscope. The excitation wavelength for TRITC phalloidin was $561 \mathrm{~nm}$.

\section{Evaluating the effect of KmPFD4 overexpression on anaerobic fermentation}

YSY005 and YSY007 were cultivated in $5 \mathrm{~mL}$ of YPD at $42{ }^{\circ} \mathrm{C}$ and shaken at $250 \mathrm{rpm}$ overnight. The precultures were then inoculated into $20 \mathrm{~mL}$ of YPD medium containing $100 \mathrm{~g} / \mathrm{L}$ glucose, $1.9 \mathrm{~g} / \mathrm{L}$ acetic acid, $0.95 \mathrm{~g} / \mathrm{L}$ furfurals, and $0.19 \mathrm{~g} / \mathrm{L}$ phenols (four phenols with the same concentration) in anaerobic bottles. The initial cells density was $\mathrm{OD}_{600}=0.3$, and the growth $\left(\mathrm{OD}_{600}\right)$, glucose consumption, and ethanol production were measured during the fermentation process.

\section{Analytical methods}

The growth of yeast was examined by measuring the $\mathrm{OD}_{600}$. Glucose and ethanol were quantified by high-performance liquid chromatography with a ROA-Organic Acid $\mathrm{H}^{+}(8 \%)$ column (Phenomenex, USA). The mobile phase was $0.0025 \mathrm{M} \mathrm{H}_{2} \mathrm{SO}_{4}$ at a column temperature of $75^{\circ} \mathrm{C}$ and a flow-rate of $0.3 \mathrm{~mL} / \mathrm{min}$.

\section{Statistical analysis}

All experiments were repeated for three times and the standard error of the mean was marked as error bars in figures.

\section{Results and discussions KmPFD4 gene cloning}

A 1590 bp DNA fragment of KmPFD4 gene including $600 \mathrm{bp}$ upstream and $600 \mathrm{bp}$ downstream of ORF was amplified from the genomic DNA of $K$. marxianus YHJ010 (Additional file 2: Fig. S2). The ORF encoded a protein of 129 amino acid residues. The amino acid sequence of KmPFD4 was of $87.60 \%, 69.77 \%$, and $55.38 \%$ identity to PFD4 from K. lactis, S. cerevisiae, and Candida albicans, respectively (Additional file 2: Fig. S2).

\section{KmPFD4 disruption, complementary, and overexpression strains were obtained}

KmPFD4 disrupted strain YSY003, KmPFD4 disrupted and retro-complementary strain YSY004, KmPFD4 overexpression strain YSY005, and KmPFD4 non-disruption control strain YSY007 were obtained. For a fair comparison in the following investigations, these strains were the same auxotroph (leu2). Figure 2 shows the confirmation results of PCR with genomic DNA as the template. The KmPFD4 in YSY007 (Table 2) was unmodified, and a $1.6 \mathrm{~kb}$ DNA fragment was amplified with primers KmPFD4-F and KmPFD4-R (Fig. 2). In YSY003, part of the ORF of KmPFD4 was substituted with a $2.2 \mathrm{~kb}$ DNA (ScURA3 expression cassette), and only a $3.4 \mathrm{~kb}$ DNA

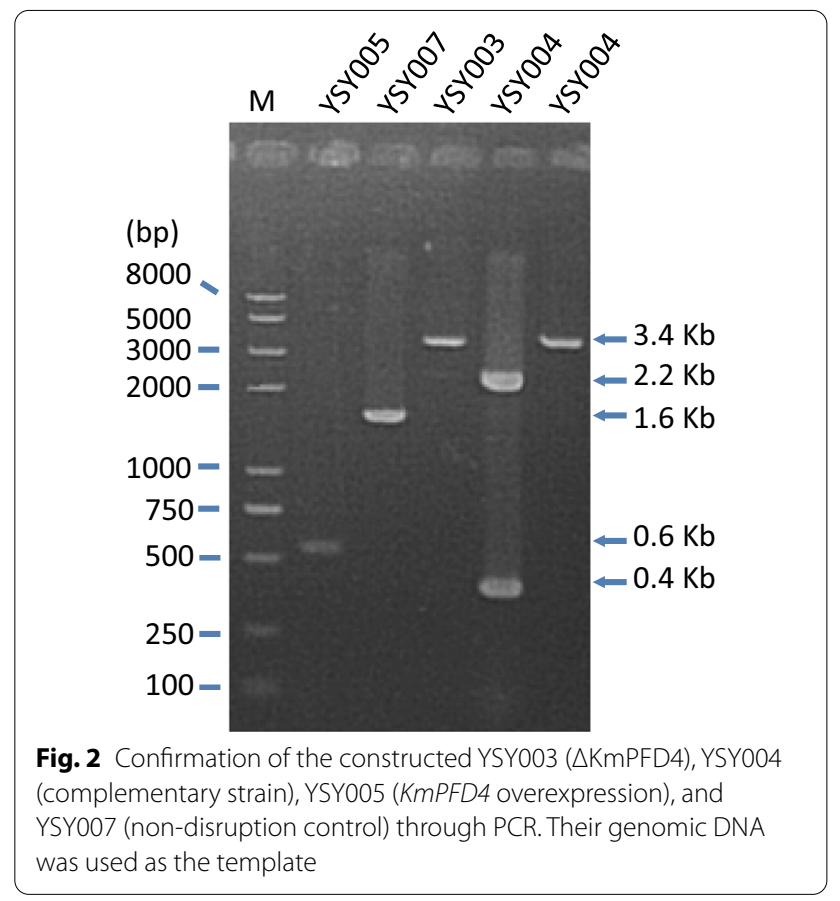

portion was amplified with primers KmPFD4-F and KmPFD4-R. If KmPFD4 was not disrupted, a $1.6 \mathrm{~kb}$ DNA was expected to be observed. YSY005 was an overexpression strain of KmPFD4. To differentiate the transformed KmPFD4 and original KmPFD4, a primer based on the terminator of the overexpression cassette (TDH3-TerR) was used with primer KmPFD4-EcoRI-F to amplify the overexpressed KmPFD4. A $0.6 \mathrm{~kb}$ fragment, which including $0.4 \mathrm{~kb}$ ORF and $0.2 \mathrm{~kb}$ terminator, was amplified (Fig. 2). YSY004 was constructed for overexpressing KmPFD4 in the KmPFD4 disrupted strain to complement the disruption of KmPFD4. Using the primers for the disruption cassette (KmPFD4-F and KmPFD4-R), a $3.4 \mathrm{~kb}$ DNA fragment was detected. Using the KmPFD4 ORF primers KmPFD4-EcoRI-F and KmPFD4-NotI$\mathrm{R}$, a disrupted KmPFD4 $(2.2 \mathrm{~Kb})$, and a wild-type KmPFD4 (0.4 Kb) were detected (Fig. 2). The expression of KmPFD4 in YSY003, YSY004, YSY005, and YSY007 strains were verified through RT-PCR. With the expression of $A C T 1$ as internal control, the relative expression levels $\left(2^{-\Delta \Delta \mathrm{Ct}}\right)$ were $0.00,0.17,0.20$, and 0.04 , respectively (Additional file 2: Fig. S3). The very low expression level of KmPFD4 in YSY003 indicated that KmPFD4 was disrupted. And the higher expression level of KmPFD4 in YSY004 and YSY005 than YSY007 indicated that the KmPFD4 was overexpressed and the disruption was complemented. Therefore, all obtained strains were correct. The expression of KmPFD4 in YSY005 was 5.15fold higher than that in YSY007 without inhibitors. Whereas, with the presence of inhibitors, the expression 
of KmPFD4 in YSY005 was 19.17-fold higher than that in YSY007.

\section{The effect of KmPFD4 disruption or overexpression on inhibitor tolerance}

During the pretreatment of lignocellulosic biomass, chemicals which inhibit cellulolytic enzyme and microbial growth and fermentation were produced. Different pretreatments produce different inhibitors in various ratios $[6,16]$. Thus, it is difficult to set defined inhibitors composition in the inhibitor evaluation study. $K$ marxianus is reported that it has higher tolerance to single kind inhibitor than S. cerevisiae. It completely assimilated furfural and vanillin in 8 and $16 \mathrm{~h}$ at an initial concentration of $2 \mathrm{~g} / \mathrm{L}$, respectively. Whereas, $S$. cerevisiae showed a lag assimilation period of 24 and $30 \mathrm{~h}$, respectively [28]. In this study, a simplified inhibitors mixture was used to evaluate the effect of genetic modification on inhibitor tolerance. Furfural and HMF were selected as the furan compounds. Since formic and levulinic acids are degradation products of furan compounds [8], they were not added to the inhibitor mixture. Acetic acid was used as a weak acid. Because the $\mathrm{pH}$ of medium was adjusted to 6.0 , the acetic acid existed as acetate. The phenolic compounds were more complicated, and 4-hydroxybenzaldehyde, syringaldehyde, catechol, and vanillin were included in the mixed inhibitors because they are the main phenols in lignocellulosic biomass hydrolysate [4, 14]. As the pretreated lignocellulosic biomass contains too many inhibitors, not all inhibitors were added to the medium to evaluate the yeast tolerance in many cases. Unrean et al. [11] added only acetate and furfural to the medium in their mechanistic analysis of inhibitor tolerance. Binary combinations of inhibitors were used in another inhibitor tolerance analysis [14]. Since inhibitors synergistically inhibit yeast growth and fermentation $[14,46]$, studies on tolerance of multiple inhibitors are more important. In this study, the mixture of inhibitors included three types of inhibitors which mimic the inhibitors in pretreated lignocellulosic biomass.

Disruption of KmPFD4 reduced the tolerance of $K$. marxianus to inhibitors. For fairly comparing the growth of each strain, YSY007 was used as control strain due to the same auxotroph (leu2) of YSY003, YS004, YSY005, and YSY007. Without inhibitors, the growth of KmPFD4disrupted strain YSY003 was slightly weaker than that of the wild-type strain YSY007, and the biomass produced was similar (Fig. 3a). The final cell density $\left(\mathrm{OD}_{600}\right)$ of each strain was approximately 20. The growth of YSY003 was apparently weaker than that of YSY007 when the medium contained inhibitors. When the medium contained mixed inhibitors, YSY003 did not grow, whereas YSY007 grew with a $24 \mathrm{~h}$ lag phase and the final cell density was only $9.57\left(\mathrm{OD}_{600}\right)$ after $39 \mathrm{~h}$ of cultivation (Fig. 3b). When YSY003 was cultivated in a medium containing a single inhibitor, it had a longer lag phase and lower biomass production than YSY007 (Fig. 3c-e). The lag phases of YSY003 in the presence of acetate sodium (Fig. 3c), furfurals (Fig. 3d) and phenols (Fig. 3e) were 3, 12 , and $15 \mathrm{~h}$, respectively, whereas the lag phases of other strains were similar with each other, approximately 3,7 , and $7 \mathrm{~h}$, respectively. The final cell densities of YSY003 in the presence of acetate sodium (Fig. 3c), furfurals (Fig. 3d) and phenols (Fig. 3e) were 10.28, 7.35, and 16.56 $\left(\mathrm{OD}_{600}\right)$, respectively. The final cell densities of the other strains were also similar, approximately 11,20 , and 22 $\left(\mathrm{OD}_{600}\right)$, respectively (Fig. 3c-e).

Overexpression of KmPFD4 rescued the weak growth of YSY003 and enhanced the tolerance to mixed inhibitors. The growth of complementary strain YSY004 in the medium without inhibitors was similar to that of YSY007 (Fig. 3a) and stronger than YSY003 in the presence of a single inhibitor (acetic acid, furfurals, or phenols) (Fig. 3c-e). Furthermore, YSY004 grew better than YSY007 in the presence of mixed inhibitors with a $12 \mathrm{~h}$ lag phase and final cell density of $15.40\left(\mathrm{OD}_{600}\right)$ (Fig. 3b).

The growth of the overexpression strain YSY005 indicated that overexpression of KmPFD4 improved the tolerance to multiple inhibitors. YSY005 showed similar results to YSY004 in the medium with a single inhibitor and grew better than YSY004 with a final cell density of $17.19\left(\mathrm{OD}_{600}\right)$ in the presence of multiple inhibitors (Fig. 3b).

To show the effect directly, the effect on tolerance to inhibitors was also evaluated on the YPD plate containing various inhibitors (Fig. 4). The growth of YSY003 was slightly weaker than that of the other strains without the inhibitors (Fig. 4a), which was consistent with the results of liquid culture. In the presence of inhibitors, the growth of YSY003 was weaker than that of other strains, especially with the presence of mixed inhibitors or phenols, the growth decreased obviously. In the presence of furfurals, all strains grew worse than those on the plates without inhibitors, and the difference in growth between YSY003 and other strains was not obvious. These results were consistent with the growth curve results (Fig. 3c). Therefore, the disruption of KmPFD4 may have less effect on the furfural tolerance of $K$. marxianus.

The overexpression of KmPFD4 rescued the deficient growth of gene disruption on the plate containing inhibitors. However, the difference in growth among the overexpression strain YSY005, complementary strain YSY004, and control strain YSY007 was not obvious on solid medium (Fig. 4).

Very high or very low concentrations of inhibitors would eliminate the difference in tolerance (all would 

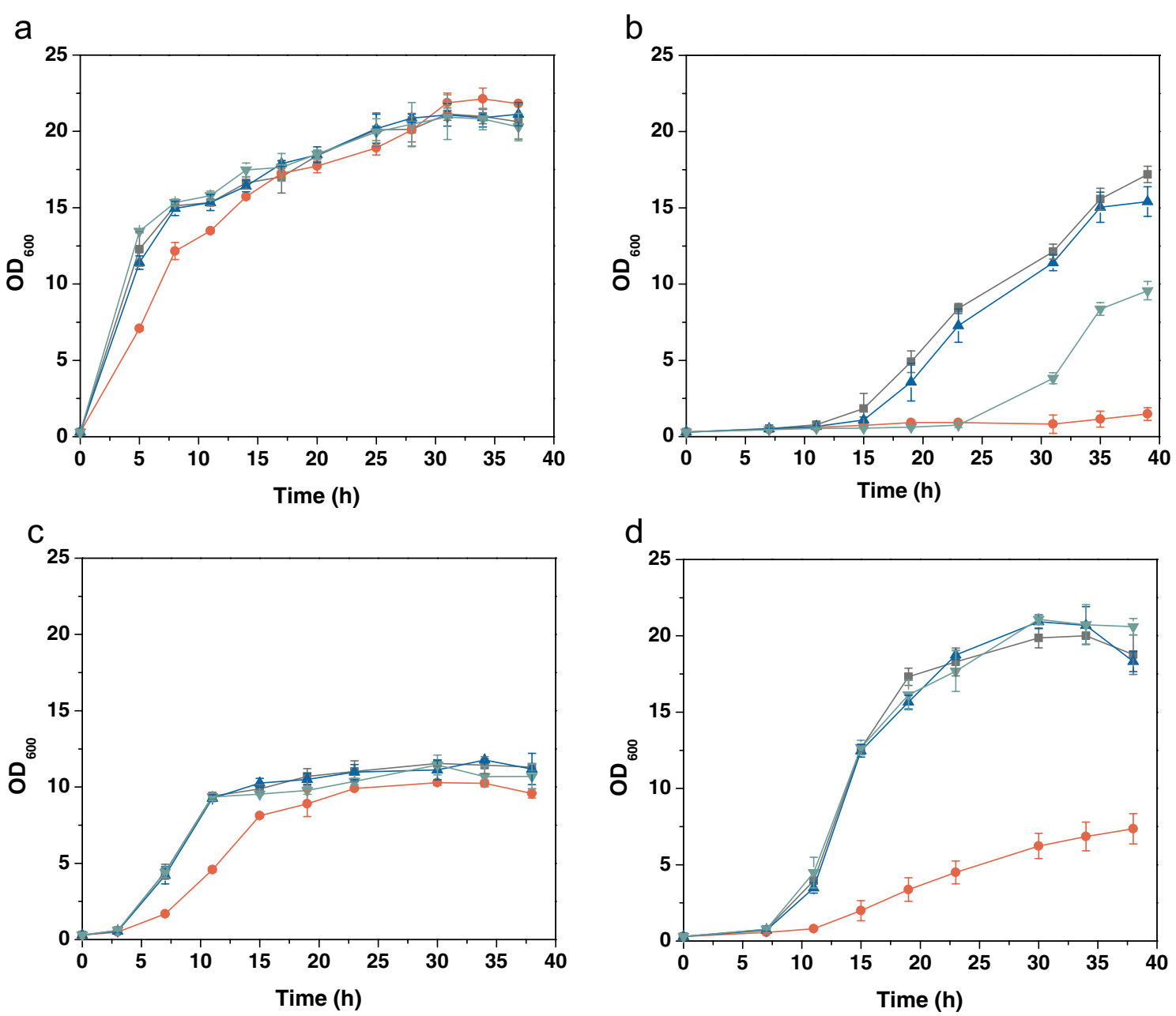

e
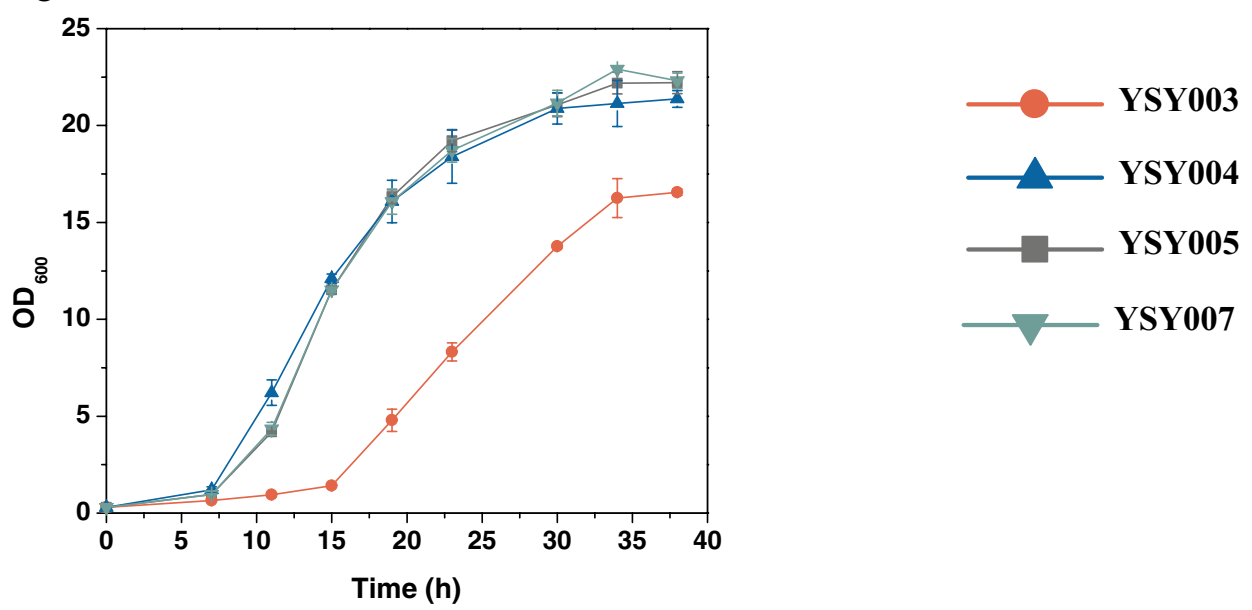

Fig. 3 The effect of KmPFD4 disruption or overexpression on tolerance to lignocellulosic biomass-derived inhibitors. YPD medium containing no inhibitor (a), mixed inhibitors (b), acetate sodium (c), furfurals (d), or phenols (e) was used to evaluate growth. All values are the means of three biological replicates \pm standard deviation at each of the time points 


\begin{tabular}{|c|c|c|c|c|c|}
\hline & $\mathbf{a}$ & $\mathbf{b}$ & c & d & $\mathbf{e}$ \\
\hline YSY005 & $\begin{array}{l}000 \% \\
000 \approx \\
000 \% \\
000 \% \\
00 \% \\
00 \% \approx \\
000 \% \\
00 \%\end{array}$ & 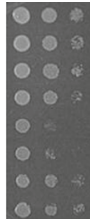 & 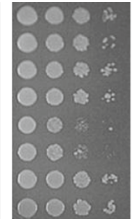 & $\begin{array}{l}05: \\
89 \\
09 \\
0 \% \\
0 \% \\
09:\end{array}$ & $\begin{array}{l}000 \\
000 \\
000 \\
009: \\
00 \\
00 \\
000 \\
00\end{array}$ \\
\hline \multicolumn{6}{|c|}{$\begin{array}{l}\text { Fig. } 4 \text { Spot assay of various strains on YPD plates containing no or } \\
\text { various inhibitors. a No inhibitor, } \mathbf{b} \text { mixed inhibitors, } \mathbf{c} \text { acetic sodium, } \\
\mathbf{d} \text { furfurals, and e phenols. Two lines of each strain were determined. } \\
\text { The concentrations of spotted cells were } 6 \times 10^{-1}, 6 \times 10^{-2}, 6 \times 10^{-3} \text {, } \\
\text { and } 6 \times 10^{-4}\left(\mathrm{OD}_{600}\right) \text {, respectively }\end{array}$} \\
\hline
\end{tabular}

grow well or not at all) (data not shown). Therefore, the concentration of inhibitors used in this study was adjusted to reflect the difference in tolerance. Therefore, the concentration of single inhibitor was different to that in mixed inhibitors. It is not comparable between different single-type inhibitors. Since gene expression and metabolic regulation are different, the difference in tolerance to inhibitors in liquid medium or on solid plates can be expected.

It was not expected that overexpression of KmPFD4 would enhance the tolerance to mixed inhibitors, whereas no enhancement was detected in tolerance to each type of inhibitor. Since multiple inhibitors can hinder microbial growth and fermentation synergistically $[14,46]$, the mixed inhibitors led to severe stress on yeast cells. It is possible that the native KmPFD4 expression level is sufficient for a single type of inhibitor tolerance. Therefore, the overexpression of KmPFD4 did not lead to increased tolerance to a single kind of inhibitors. In addition, KmPFD4 was not effective for all inhibitors. Finally, the concentration of single inhibitor may not be high enough to make a difference of tolerance between overexpression and control strains. Therefore, overexpression of KmPFD4 only showed apparent tolerance to mixed inhibitors. As a result, YSY003 did not grow with the presence of mixed inhibitors while other strains had a longer lag phase than with the presence of single type of inhibitor (Fig. 3b).

\section{The disruption of KmPFD4 disturbed the cytoskeleton assembly}

Actin and tubulin are the main components of the cytoskeleton, and cytoplasmic prefoldin is important for the folding of actin and tubulin monomers during cytoskeleton assembly [29]. Therefore, the effect of KmPFD4 disruption on the cytoskeleton in K. marxianus was determined.

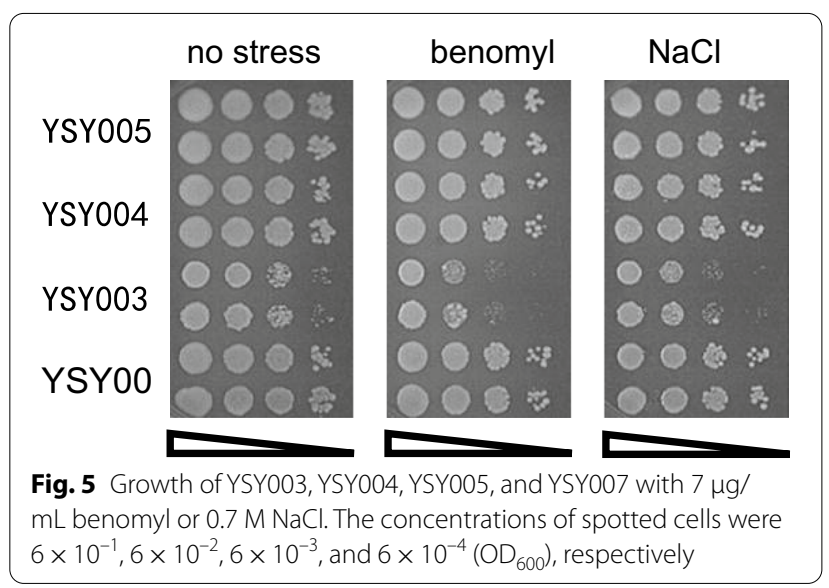

First, the effect of KmPFD4 disruption on tubulin was evaluated. As shown in Fig. 5, the growth of YSY003 (KmPFD4-disrupted) was weaker than that of other strains on the plate containing benomyl or $\mathrm{NaCl}$. These results indicated that the disruption of KmPFD4 increased the sensitivity of the cells to benomyl, which interfered with tubulin assembly. In addition, disruption led to increased sensitivity of the cells to osmotic pressure $(0.7 \mathrm{M} \mathrm{NaCl})$. Disruption of KmPFD4 may reduce the self-repair ability of cells to microtubule disorder, which is driven by inhibitors. In S. cerevisiae, PFD4 disruption leads to increased sensitivity to benomyl, although the difference is not so apparent with $\mathrm{NaCl}$ [45]. This is possibly due to the different yeasts or the function of PFD4 in different yeasts.

Second, the effect of KmPFD4 disruption on actin was evaluated. As shown in Fig. 6, F-actin was stained with TRITC-phalloidin. Without inhibitors, the F-actins in most cells of YSY004, YSY005, and YSY007 existed as polarized patches $(86.5 \%, 88.9 \%$, and $86.9 \%$, respectively), while polarized actins decreased (54.2\%, 55.6\%, and $47.7 \%$ respectively) in the presence of inhibitors (Fig. 6). In contrast, in the KmPFD4-disrupted strain YSY003, F-actin in most cells was depolarized even without inhibitors (23.2\% polarized), and the ratio of the cells containing polarized actin decreased to $13.2 \%$ in the presence of inhibitors. It is possible that the disruption of KmPFD4 interfered with the assembly of actin and led to depolarization (Fig. 6).

KmPFD4 disruption also changed the cells shape of the $K$. marxianus. As shown in Fig. 6, the average length, width, and ratio of KmPFD4-disrupted cells (YSY003) were $4.00 \mu \mathrm{m}, 3.47 \mu \mathrm{m}$ and 1.17, whereas the length, width, and ratio of control cells (YSY007) were $4.01 \mu \mathrm{m}, 2.46 \mu \mathrm{m}$ and 1.65 , respectively. Since the shape of cells was related to the cytoskeleton, these results 

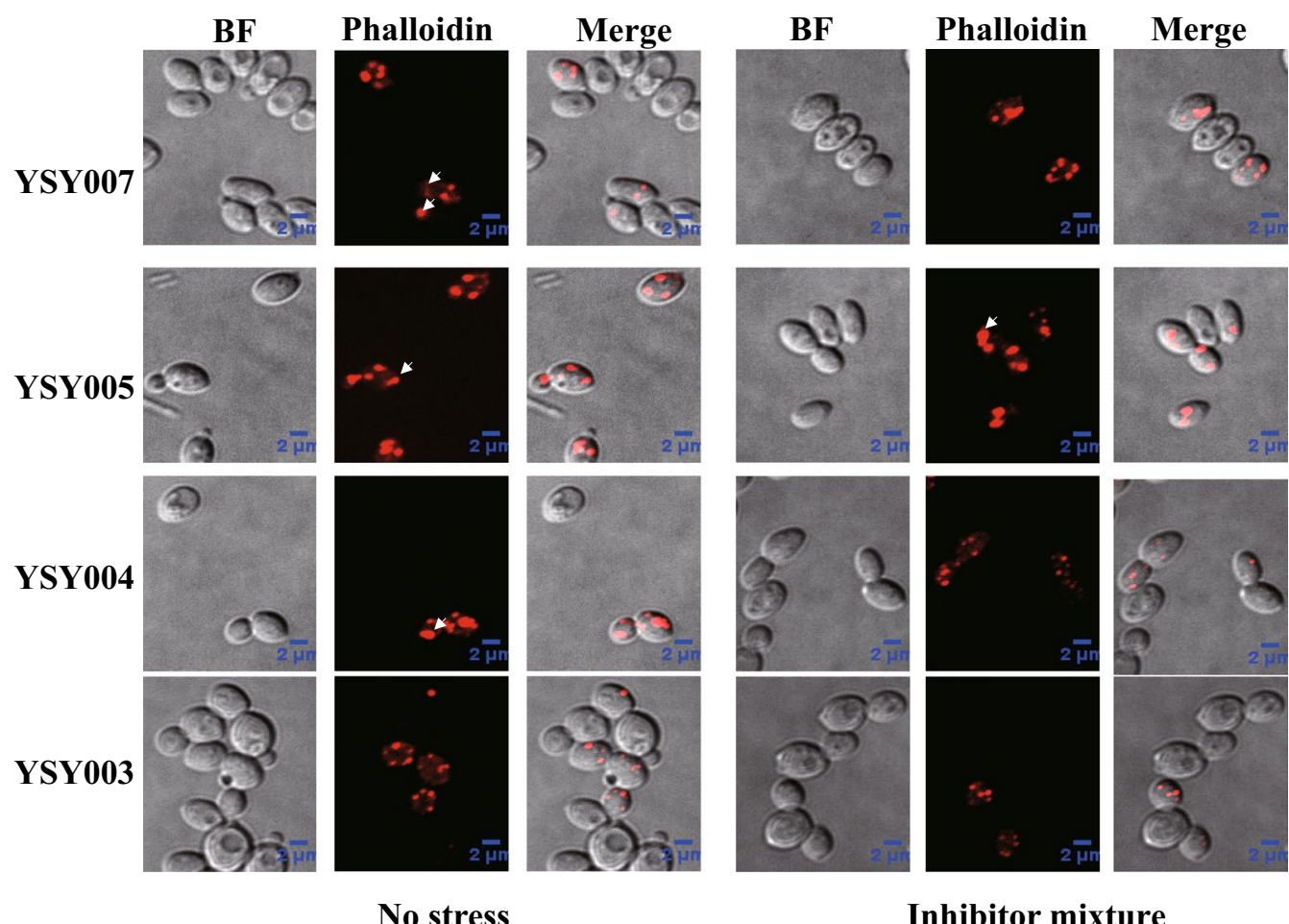

No stress
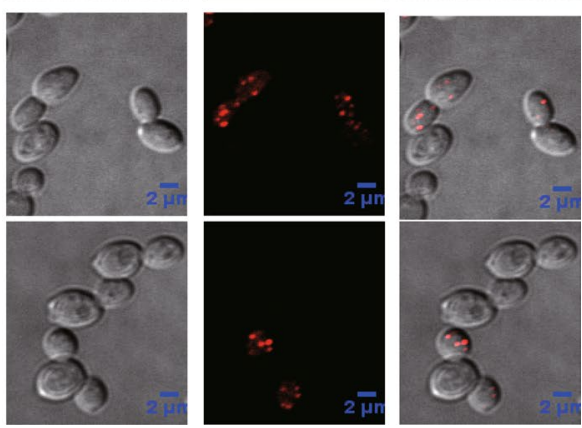

Inhibitor mixture

Fig. 6 TRITC-phalloidin staining of YSY003, YSY004, YSY005, and YSY007 F-actin with or without the presence of mixed inhibitors. The white arrows indicate the patches or cables of F-actin

also indicated that the disruption of KmPFD4 interfered with the cytoskeleton assembly.

There are almost no reports connecting the tolerance of lignocellulosic biomass-derived inhibitors to prefoldin and the cytoskeleton. Consistent with the role of prefoldin in actin and tubulin folding, the deletion of prefoldin-encoding genes in S. cerevisiae results in impaired cytoskeleton functions, although none of the prefoldin subunits are essential for yeast viability [29]. In this study, the presence of inhibitors led to the disturbation of F-actin in the control strain, indicating that the cell skeleton is a possible target of inhibitors. In another study, the actin cytoskeleton was thought to be a cellular target for oxidative stress [47]. Lignocellulosic biomassderived inhibitors suppress yeast growth and viability by producing stresses, such as ROS and membrane permeability. The accumulation of ROS leads to damage of the mitochondria and vacuole membranes, actin cytoskeleton, and nuclear chromatin $[12,48]$. The prefoldins help the assembly of actin and tubulin, and the disruption of the prefoldin gene may reduce cytoskeleton assembly efficiency and reduce tolerance to stress. Therefore, the inhibitors disturbed the actin cytoskeleton structure and reduced cell viability. The disruption of KmPFD4 led to disruption of the actin cytoskeleton assembly. Thus, the actin cytoskeleton would be destroyed further in the presence of inhibitors. As a result, the inhibitor tolerance ability decreased. Overexpression of KmPFD4 may enhance the repair ability of the cytoskeleton through prefoldin and improve the inhibitor tolerance. In this study, the cytoskeleton disorder caused by disruption of KmPFD4 is a possible reason for decreased inhibitor tolerance.

\section{The intracellular ROS concentration increased} with the presence of inhibitors in the KmPFD4-disrupted strain

As lignocellulosic biomass-derived inhibitors produce ROS during their catabolism $[12,48]$, the overproduced ROS should be degraded to avoid harm to yeast cells. The ROS level of YSY003, YSY004, YSY005, and YSY007 with the presence of inhibitors were determined (Fig. 7). Without inhibitors, the intracellular level of ROS in each strain was similar, although the ROS level of YSY003 was slightly higher than that of the other strains. In the presence of mixed inhibitors, the ROS concentration of all strains increased, and the intracellular ROS level of YSY003 was 1.52-, 1.60-, 1.37-fold higher than that of the YSY007, YSY004, YSY005 (Fig. 7). It was unexpected that the ROS level of KmPFD4 overexpression strain YSY005, 


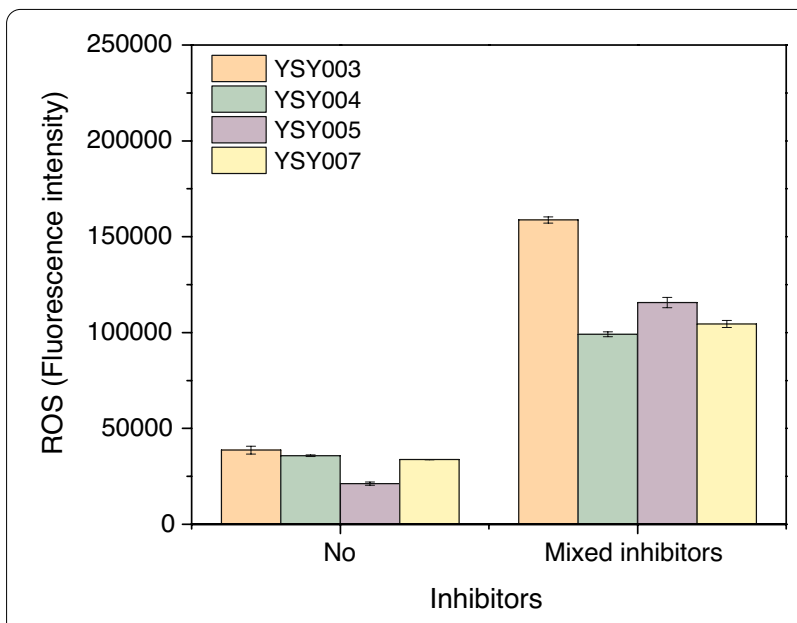

Fig. 7 Disruption of KMPFD4 led to greater ROS accumulation with the presence of mixed inhibitors. All values are the means of three biological replicates \pm standard deviation at each of the time points

which grow best with the presence of inhibitors (Fig. 3b) was not the lowest. Though we repeated the experiment, the results were similar. The reason is not clear. The PFD4 (GIM3) disruption in S. cerevisiae led to greater sensitivity to $\mathrm{H}_{2} \mathrm{O}_{2}$ compared with the disruption of other prefoldin subunits; however, the disruption of PFD2, 5, and 6 [45] did not change the sensitivity to $\mathrm{H}_{2} \mathrm{O}_{2}$. Therefore, it is possible that KmPFD4 is important for the removal of superoxide or hyperoxide, which is a component of ROS. However, prefoldins are thought to be co-chaperones in protein folding, especially in cytoskeleton assembly. Why disruption of KmPFD4 led to ROS accumulation was not clear. Therefore, subcellular localization and transcriptome analysis were conducted.

\section{KmPFD4 was localized in both the nucleus and cytoplasm in the presence of inhibitors}

After the effects of disruption and overexpression of KmPFD4 on tolerance to inhibitors were evaluated, the intracellular location of PFD4 was determined to identify the possible mechanism of inhibitor tolerance. YSY008 was the control strain that only expressed EGFP, and YSY009 expressed the fusion protein KmPFD4-EGFP. Without inhibitors, EGFP was distributed in the cytoplasm of YSY008, whereas the green fluorescence of fusion protein in YSY009 cells was focused in or near the nucleus, which was stained with DAPI (blue) (Fig. 8a). These results indicated that KmPFD4 could enter the nucleus or accumulate around it. In the presence of inhibitors in the medium, KmPFD4 was distributed in both the nucleus and cytoplasm, although the fluorescence in the nucleus was stronger (Fig. 8b).

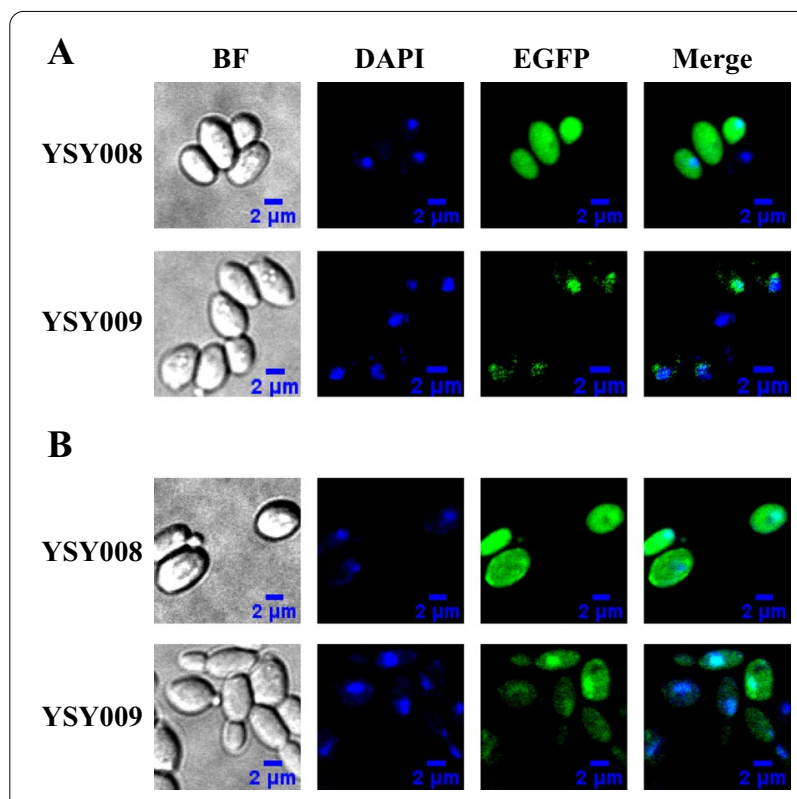

Fig. 8 The intracellular localization of KmPFD4. a Without inhibitors, and $\mathbf{b}$ with mixed inhibitors. YSY008, expressing EGFP; YSY009, expressing KmPFD4-EGFP

In eukaryotes, the prefoldins are localized to both the cytoplasm and nucleus [30]. In the cytoplasm, the prefoldins are mainly related to actin and tubulin assembly and in the nucleus, they regulate gene expression (reviewed by Laura Payán-Bravo [30]).

In this study, KmPFD4 was mostly localized near or within the nucleus, while in the presence of inhibitors, some KmPFD4 was transported to the cytoplasm. It is possible that the inhibitors disturbed the structure of the cytoskeleton, then the prefoldin including KmPFD4 moved to the cytoplasm to repair the damage. Furthermore, KmPFD4 may regulate gene expression due to its localization to the nucleus. However, the gene expression regulation function of KmPFD4 has seldom been reported. Therefore, the transcriptome of the KmPFD4disrupted strain was analyzed.

\section{Transcriptomic analysis of the KmPFD4-disrupted strain} with multiple inhibitors indicated that KmPFD4 regulated the gene expressions required for inhibitor tolerance As KmPFD4 is located in or near the nucleus, the transcriptome was used to verify whether the disruption of KmPFD4 changed the gene expression. We conducted transcriptomic analysis of $K$. marxianus YSY003 (KmPFD4-disrupted) and YSY007 (no disruption of KmPFD4) with or without lignocellulosic biomassderived inhibitors by using RNA-seq. The levels of gene expression, normalized as TPM, were applied to the FCs of the DEGs (with absolute FCs $\geq 2 ; p<0.001$ ). The 
RNA-seq results were included in the following two relevant pairwise comparisons of gene expression levels: YSY007-I vs. YSY007-C (with vs. without inhibitors), and YSY003-I vs. YSY003-C (with vs. without inhibitors). Almost no read of KmPFD4 was detected in YSY003 and this confirmed that KmPFD4 was disrupted in YSY003 (Additional file 1).

Furthermore, the expression of 12 genes was analyzed by RT-PCR for validation of RNA seq results. With the presence of the inhibitors, the expression of mitochondrial peroxiredoxin $(P R X 1)$, superoxide dismutase [Mn] $(S O D 2)$, and succinate dehydrogenase [ubiquinone] (SDH1) was up-regulated in YSY007 and down-regulated in YSY003. Though $\mathrm{Cu} / \mathrm{Zn}$ superoxide dismutase (SOD1), nicotinamide-nucleotide adenylyltransferase 1 (NMNAT), NADH pyrophosphatase (NUDC), and multicopy suppressor of SNF1 mutation (MSN2) were upregulated in both YSY007 and YSY003, the changes in YSY003 was less than that in YSY007. Peroxisomal catalase $\mathrm{A}(C T A 1)$ and citrate synthase $(C I T 1)$ were up-regulated in YSY007, and down-regulated in YSY003. ATP synthase subunit 4 (ATP4) was down-regulated in both YSY003 and YSY007. Glucose-6-phosphate isomerase (PGI1) and glyceraldehyde-3-phosphate dehydrogenase 3 (TDH3) were up-regulated in both YSY003 and YSY007. Though the expression changes of the analyzed genes were not the same as RNA-seq results, their trends of the changes in real-time PCR were similar to those in RNAseq (Additional file 2: Table S2).

Based on these results, we focused on the DEGs in $K$. marxianus YSY007 or YSY003 with or without the multiple inhibitors (I vs. C), especially those DEGs related to ROS detoxification, central carbon metabolism, mitochondrial respiratory chain, $\mathrm{NAD}(\mathrm{P})^{+}$biosynthetic enzymes, and nicotinate metabolism. There were some changes of the gene expression with the presence of inhibitors when KmPFD4 was disrupted. Though it was still difficult to make a conclusion that KmPFD4 regulated the gene expression, KmPFD4 did affect the gene expression directly or indirectly.

The genes related to ROS detoxification were not upregulated in the KmPFD4-disrupted strain in the presence of inhibitors. Since ROS is produced during the metabolism of inhibitors [12], removing over-accumulated ROS is important for inhibitor tolerance. As expected, when exposed to the stress of multiple inhibitors, most of the DEGs related to ROS detoxification were upregulated, including SOD1, SOD2, PRX1, GPX2, peroxiredoxin (HYR1), thioredoxin reductase(TRR1), glutamate-cysteine ligase (GSH1), superoxide dismutase 1 copper chaperone (CCS1), glutaredoxin-1 (GRX1), monothiol glutaredoxin-5 (GRX5), peptide methionine sulfoxide reductase (PMSR), NADPH-dependent methylglyoxal reductase (GRE2), thioredoxin-2 (TRX2), thioredoxin-3 (TRX3), and putative uncharacterized oxidoreductase gene (WCAG), while cytosolic catalase $\mathrm{T}$ (CTT1), peroxiredoxin (DOT5), glutathione S-transferase 1 (GST1), and Cys-Gly metallodipeptidase (DUG1) were downregulated in the YSY007-I vs. YSY007-C group, as previously reported [27]. However, in the KmPFD4disrupted YSY003-I vs. YSY003-C group, although the expression of GPX2, HYR1, TRR1, TRX1, and GRE2 were upregulated as in the YSY007-C vs. YSY007-I group, most genes were not significantly regulated in the YSY003-C vs. YSY003-I group (Additional file 1). These results indicated that the expression of genes involved in ROS elimination in the KmPFD4 disruption strain was relatively unchanged compared to the wild-type strain in response to the presence of inhibitors.

The disruption of KmPFD4 reduced the expression of the genes of the respiratory chain and hindered ATP generation. The mitochondrial respiratory chain forms membrane potential to produce ATP and transfers electrons in the form of multi-enzyme complexes. As shown in the additional file 1, under the stress of the multiple inhibitors, rotenone-insensitive NADH-ubiquinone oxidoreductase (NDI1), external NADH-ubiquinone oxidoreductase 1 (NDH1), SDH1/2/3/4, and ubiquinol cytochrome-c reductase complex (QCR9) were upregulated and there was no significant change in QCR1, cytochrome b-c1 complex subunit Rieske (RIP1), QCR6, and ATP4 in the control (YSY007-I vs YSY007-C) pairwise comparison. Whereas in the YSY003-I vs. YSY003$\mathrm{C}$ group, there was no significant change in NDI1 or $S D H 1 / 2 / 3 / 4$. Furthermore, QCR1, RIP1, QCR6, QCR9, and ATP4 were downregulated. The expression of the counterpart genes in the YSY003-I vs. YSY003-C group was downregulated compared with those in the YSY007I vs. YSY007-C group. These results suggested that with the stress of multiple inhibitors, disruption of KmPFD4 reduced the expression of the genes of the respiratory chain and may hinder ATP generation further. In previous studies, in the presence of inhibitors, the expression of genes related to ATP production was upregulated due to increased ATP required for inhibitors degradation and removal [26, 27]. However, in the wild-type strain, the intracellular concentration of ATP is decreased due to increased consumption [49], disruption of the proton gradient of mitochondria [50], non-specific hydrolysis of ATP [49], and decreased intracellular concentration of NADH for ATP production. Decreased genes expression in the respiratory chain led to more severe shortage of ATP. ATP is required to pump the proton in the presence of a weak acid [11]. In addition, ATP is the adenylyl-backbone donor for $\mathrm{NAD}^{+}$. The shortage of ATP is expected to reduce inhibitor tolerance. 
The genes related to $\mathrm{NAD}^{+}$biosynthetic enzymes and nicotinate metabolism were not upregulated in the KmPFD4-disrupted strain compared to the control strain. The essential coenzymes nicotinamide adenine dinucleotides, $\mathrm{NAD}(\mathrm{P})^{+}$and $\mathrm{NAD}(\mathrm{P}) \mathrm{H}$, participate in key redox reactions and contribute to maintaining cells fitness and genome stability [51]. $\mathrm{NAD}^{+}$is important in lignocellulosic biomass-derived inhibitor tolerance and is the source of total $\mathrm{NAD}(\mathrm{P})^{+}$and $\mathrm{NAD}(\mathrm{P}) \mathrm{H}$ [26]. Under the stress of the multiple inhibitors, almost all of the DEGs related to $\mathrm{NAD}^{+}$biosynthetic enzymes and related proteins were upregulated, except PNC1 encoding nicotinamidase in the YSY007-I vs. YSY007-C group. However, in the YSY003-I vs. YSY003-C group, these genes were not significantly regulated except for nucleoside transporter FUN26 which was downregulated. This may imply that the $\mathrm{NAD}^{+}$biosynthesis in the KmPFD4-disrupted strain was not enhanced as those in the wild-type strain in response to the presence of inhibitors. Therefore, the supply of $\mathrm{NAD}^{+}$in the KmPFD4-disrupted strain was not improved with the increased demand for inhibitor tolerance, and the tolerance ability was decreased.

In the presence of inhibitors, the genes related to glycolysis were up-regulated, in the KmPFD4-disrupted strain. In contrast, most of the genes related to glycolysis were down-regulated or no significant change in the non-disrupted strain which was also reported in S. cerevisiae [9]. Central carbon metabolism plays an important role in the carbon source and energy production in yeast cells. As shown in Additional File $1 \mathrm{~s}$, in the presence of multiple inhibitors, quite a few DEGs related to glycolysis/gluconeogenesis, such as PGI1, 6-phosphofructokinase subunit $(P F K 1 / 2)$, triosephosphate isomerase (TPI), TDH3, phosphoglycerate kinase $(P G K)$, enolase $(E N O)$, pyruvate kinase $(P Y K)$, Pyruvate decarboxylase $(P D C)$ etc., were upregulated in the YSY003-I vs. YSY003-C group, while there was no significant change in YSY007-I vs. YSY007$\mathrm{C}$ pairwise comparison.

On the other hand, among those DEGs in the TCA cycle, $S D H 1 / 2 / 3 / 4$ and malate dehydrogenase $(M D H 1 / 2)$ were upregulated and there was no significant change to IDP2 in YSY007-I vs. YSY007-C pairwise comparison. On the other hand, in the YSY003-I vs. YSY003-C group, there was no significant change in the expression level of $S D H 1 / 2 / 3 / 4$ and $M D H 1$. Furthermore, $M D H 2$ and IDP2 were downregulated. Likewise, isocitrate lyase (ICL1) was downregulated in YSY003-I vs. YSY003-C pairwise comparison, but there was no significant change in YSY007-I vs. YSY007-C pairwise comparison (Additional file 1). These results indicated that the disruption of KmPFD4 stimulated glycolysis/gluconeogenesis, but hindered the TCA cycle. It is well-known that the TCA cycle provides more ATP and $\mathrm{NAD}(\mathrm{P}) \mathrm{H}$, which are necessary for inhibitors removal and conversion. Our previous study have shown that the intracellular concentrations of ATP and NAD $(\mathrm{P}) \mathrm{H}$ decreased due to the consumption of inhibitors [26]. The disruption of KmPFD4 led to a severe shortage of ATP and $\mathrm{NAD}(\mathrm{P}) \mathrm{H}$ and finally reduced the inhibitor tolerance.

The results of the TTC staining indicated that the disruption of KmPFD4 decreased the respiration. On the plates, the YSY007 colonies were red, whereas, the color of YSY003 colonies was lighter than that of YSY007. And the white colonies indicated much weaker respiration. These results illustrated that the respiration of $K$. marxianus was interfered after KmPFD4 disruption (Additional file 2: Fig S4).

The expression of genes related to the MSN2/4mediated stress response element (STRE) is intricate. Transcriptional activator MSN2 in $S$. cerevisiae regulates the transcription of the genes associated with oxidative stress, heat shock, and high concentration of ethanol [52]. Because furfural and phenolic inhibitors lead to intracellular ROS accumulation [12, 13], the expression of MSN2/4 mediated stress response elements in $K$. marxianus were also analyzed through RNA-seq. MSN4 was not found in the genome of $K$. marxianus, and the KmMSN2 expression was downregulated in YSY003 with the presence of inhibitors, whereas in YSY007, its expression was upregulated (Additional file 1). Though the expression levels of several genes such as hexokinase 1 (HXK1), glycogen phosphorylase (GPH1), heat shock protein $S S A 3$, aldehyde dehydrogenase 5 (ALD5), heat shock protein 26 (HSP26) and heat shock protein 31 (HSP31) etc. were similar in both pairwise comparisons, most regulations of DEGs were different between the pairwise comparisons, and these genes also presented in ROS or central carbon metabolism groups (Additional file 1). The expression of SSA3, SOD2, TDH2, HSP26, $H S P 31, M D H 1$, and $M D H 2$ which enhance the stress tolerance was upregulated with the presence of inhibitors in YSY007. The furfural tolerance of $S$. cerevisiae is improved through overexpression of MSN2. Various kinds of antioxidant enzymes are highly expressed by the constitutive overexpression of MSN2 even under the non-stress condition, therefore, yeast cells adapt to exposure to acute oxidative stress caused by furfural [52]. In this study, the upregulated expression of MSN2 and some STRE genes in YSY007 with the presence of inhibitors indicated increased response to inhibitors and the ROS produced by these inhibitors. However, KmPFD4 disruption led to decreased response to inhibitors.

Prefoldins can regulate gene expression not only as free single subunit but also as a prefoldin complex. Canonical prefoldin interacts with specific transcription factors and modulates their activity [30]. The PFD1 [31], PFD3 [32] 
[53], PFD5 [54], PFDN1-PFDN2-PFDN5- PFDN6 complex [36], and PFD5-PFD6 complex [35] were reported that they can regulate gene expression in mammalian cells, hepatitis B, yeast, or plants. However, there have been few reports regarding PFD4 in the regulation of gene expression. Iijima et al. reported that PFD4 (C-1 protein) is with possible transcription factor activity at the $G(1)-S$ phase transition in human fibroblast cell lines [33], but no further study is reported. In S. cerevisiae, the absence of prefoldin subunits, but not the prefoldin complex, alters stress-induced transcription [45]. Amorim et al. reported that PFD4 deletion in S. cerevisiae altered $T R X 2, C T T 1$, and HSP26 transcription when cells were exposed to $\mathrm{H}_{2} \mathrm{O}_{2}$ [45]. The function of KmPFD4 in the regulation of the genes involved in the tolerance of $K$. marxianus has not been reported yet.

The transcriptome analysis results indicated that the disruption of KmPFD4 down regulated the expression of the most genes related to ATP and $\mathrm{NAD}(\mathrm{P}) \mathrm{H}$ synthesis, and the TCA cycle (Additional File 1). The expression of these genes did not respond to the presence of inhibitors after KmPFD4 was disrupted. Therefore, a greater shortage of ATP or NAD(P)H occurred, and led to decreased detoxication ability to inhibitors and reduced ROS elimination ability. Thus, the inhibitor tolerance of yeast cells was decreased.

\section{The disruption of KmPFD4 reduced the tolerance of $K$. marxianus to other stresses}

After the effect of KmPFD4 disruption on the tolerance of $K$. marxianus to lignocellulosic biomass-derived inhibitors was determined, its effect on ethanol, temperature, and osmotic stress was evaluated by measuring their growth (Fig. 9). Therefore, the disruption of KmPFD4 not only reduced the inhibitor tolerance of $K$. marxianus, but also reduced their tolerance to other stresses.

Microtubules and tubulin are important for cell survival and proliferation in glucose-starved non-small cell lung cancer cells [55], chemotherapy-resistant tumor cells [56] and mammalian cells exposed to $\mathrm{H}_{2} \mathrm{O}_{2}$ stress [57]. They are also correlated with arsenic resistance in S. cerevisiae [58], the response to heat stress in S. cerevisiae [59] and hydrostatic pressure tolerance in Schizosaccharomyces pombe [60]. Increase of external osmolarity caused loss of actin filament cable redistribution of cortical actin filament patches [61]. The actin cytoskeleton is a cellular target for oxidative stress [47]. High concentration of ethanol impaired cellular wall permeability by disrupting sorting and signaling functions and provoking an increase in cell size, which caused a cell cycle delay. This correlates with the disappearance of actin cables and the diffusion of actin patches in yeast cells [62, 63]. Since KmPFD4 is one of the units of prefoldin that assists the assembly of the cytoskeleton, it is expected that KmPFD4 is important in stress other than lignocellulosic biomass-derived inhibitors. However, the overexpression of KmPFD4 did not improve tolerance to these stresses. It is possible that osmotic, ethanol, and temperature stresses are not strongly related to ROS. Thus, the function of KmPFD4 in the expression of genes related to ROS was not reflected.

\section{Overexpression of KmPFD4 improved the fermentation ability of $K$. marxianus}

As overexpression of KmPFD4 improved the tolerance of $K$. marxianus to mixed inhibitors, ethanol fermentation from glucose in the presence of mixed inhibitors was also determined. From $100 \mathrm{~g} / \mathrm{L}$ glucose, YSY005 produced $35.11 \mathrm{~g} / \mathrm{L}$ ethanol in $36 \mathrm{~h}$ with a productivity of $0.98 \mathrm{~g} /$ (L.h), whereas YSY007 only produced $32.23 \mathrm{~g} / \mathrm{L}$ ethanol in $48 \mathrm{~h}$ with a productivity of $0.67 \mathrm{~g} /(\mathrm{L} \cdot \mathrm{h})$ (Fig. 10). Ethanol production of YSY005 increased only $8.93 \%$ $(\mathrm{P}$ value $=0.082)$, whereas, productivity of ethanol improved by $46.27 \%$ ( $\mathrm{P}$ value $=0.022$ ). These results indicated that overexpression of KmPFD4 increased ethanol fermentation ability, especially in productivity with the presence of inhibitors. Since overexpression of KmPFD4 did not improve ethanol tolerance obviously (Fig. 9d), it is possible that increased tolerance to inhibitors led to better growth, resulting in high ethanol productivity. The ethanol yield of YSY005 and YSY007 were $0.40 \mathrm{~g} / \mathrm{g}$ and $0.38 \mathrm{~g} / \mathrm{g}$ of consumed glucose, respectively. It is about $78.27 \%$ and $74.36 \%$ of theoretical yield $(0.511 \mathrm{~g} / \mathrm{g})$. Overexpression of KmPFD4 only improved $6.83 \%$ of the yield $(\mathrm{P}$ value $=0.048)$. These results were consistent with other reports that the inhibitors delayed yeast fermentation and did not significantly reduce the ethanol yield $[64,65]$. Therefore, the improvement in ethanol production and yield was not as obvious as that in productivity through the overexpression of KmPFD4.

\section{Conclusions}

KmPFD4 affects the tolerance of $K$. marxianus to lignocellulosic biomass-derived inhibitors by maintaining the structure of the cytoskeleton and regulating the genes expression in response to ROS elimination and inhibitors degradation. Disruption of KmPFD4 led to a more severely disordered cytoskeleton and reduced the viability of the cells in the presence of inhibitors. Furthermore, the disruption of KmPFD4 led to a decrease in genes expression in response to the presence of inhibitors though the mechanism of KmPFD4 affecting gene expression needs further study. Thus, the supply of ATP and $\mathrm{NAD}(\mathrm{P}) \mathrm{H}$ required for inhibitor tolerance is decreased. Overexpression of KmPFD4 enhanced the restoration of the disturbed cytoskeleton and improved 

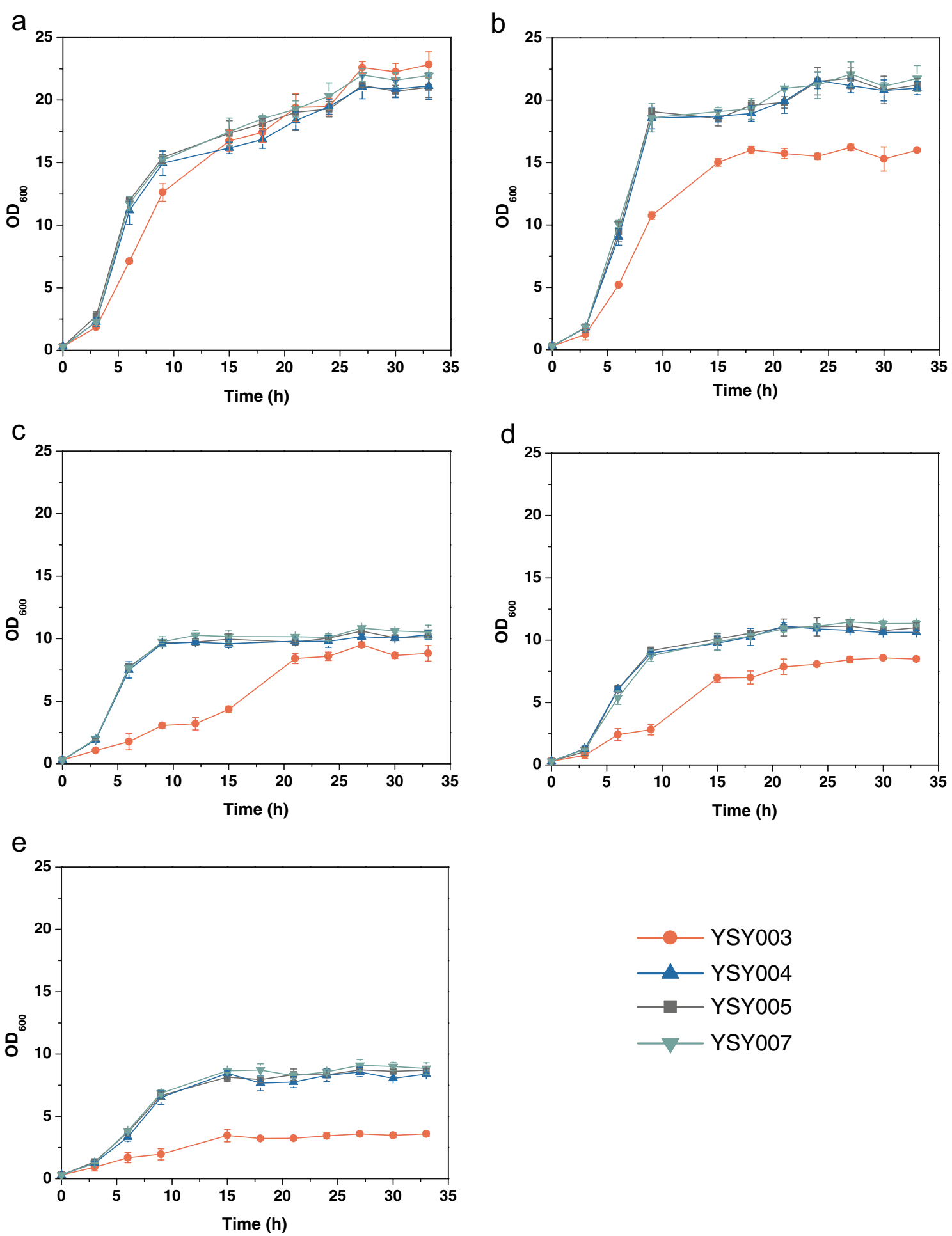

Fig. 9 Disruption of KmPFD4 reduced the tolerance of $K$. marxianus to various stresses. a No stress, $\mathbf{b} 180 \mathrm{~g} / \mathrm{L} \mathrm{glucose,} \mathbf{c} 0.7 \mathrm{M} \mathrm{NaCl}$, d $20 \mathrm{~g} / \mathrm{L}$ ethanol, and $\mathbf{e} 45^{\circ} \mathrm{C}$. All values are the means of three biological replicates \pm standard deviation at each of the time points 

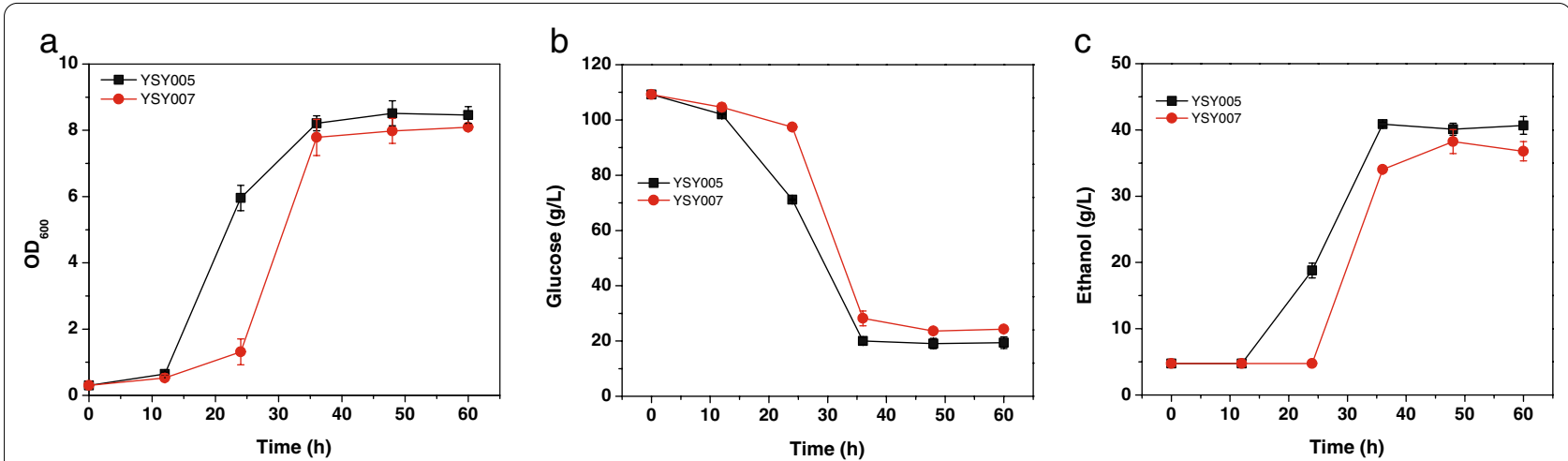

Fig. 10 Anaerobic fermentation with mixed inhibitors using KmPFD4 overexpression strain YSY005 and control strain YSY007. a Growth, b glucose consumption, and $\mathbf{c}$ ethanol production. All values are the means of three biological replicates \pm standard deviation at each of the time points

inhibitor tolerance. Therefore, KmPFD4 affects the tolerance to inhibitors in improving the cytoskeleton assembly and related gene expression. The study on KmPFD4 is related to a wide spectrum of stress tolerance, which provides a potential route to improve the tolerance of yeast to multiple lignocellulosic biomass-derived inhibitors.

\section{Supplementary Information}

The online version contains supplementary material available at https://doi. org/10.1186/s12934-021-01715-y.

Additional file 1. Functional categories and fold changes of comparative expression (in form of $\log _{2} \mathrm{FC}$ ) of differentially expressed genes in tolerance to multiple inhibitors.

Additional file 2. Additional figures and tables.

\section{Acknowledgements}

The authors acknowledge some devices supports from the Core facility center for life science in USTC.

\section{Authors' contributions}

NNZ, YYS, and FEW performed experiments, processed data. JH conceived and designed the experiments, wrote the manuscript. DMW conducted the transcriptome analysis and revised the manuscript. All authors read and approved the final manuscript.

\section{Funding}

This work was supported by the National Natural Science Foundation of China (31770085 and 32071469), Tianjin Synthetic Biotechnology Innovation Capacity Improvement Project (TSBICIP-KJGG-007), and National Key R\&D Program of China (2020YFC1316400).

\section{Availability of data and materials}

The dataset supporting the conclusions of this article are included within the article and its additional files.

\section{Declarations}

Ethics approval and consent to participate

Not applicable.

\section{Consent for publication}

Not applicable.

\section{Competing interests}

The authors declare that they have no competing interests.

\section{Author details}

${ }^{1}$ School of Life Sciences, University of Science and Technology of China, Hefei 230027, Anhui, People's Republic of China. ${ }^{2}$ Hefei National Laboratory for Physical Science at the Microscale, Hefei, Anhui 230026, People's Republic of China. ${ }^{3}$ Biomedical Sciences and Health Laboratory of Anhui Province, University of Science \& Technology of China, Hefei 230027, China.

Received: 18 April 2021 Accepted: 2 December 2021

Published online: 14 December 2021

\section{References}

1. Soltanian S, Aghbashlo M, Almasi F, Hosseinzadeh-Bandbafha H, Nizami AS, OK YS, Lam SS, Tabatabaei M. A critical review of the effects of pretreatment methods on the exergetic aspects of lignocellulosic biofuels. Energ Convers Manage. 2020;212:1.

2. Ren NQ, Wang AJ, Cao GL, Xu JF, Gao LF. Bioconversion of lignocellulosic biomass to hydrogen: potential and challenges. Biotechnol Adv. 2009:27:1051-60.

3. Valentine J, Clifton-Brown J, Hastings A, Robson P, Allison G, Smith P. Food vs fuel: the use of land for lignocellulosic next generation' energy crops that minimize competition with primary food production. GCB Bioenergy. 2012:4:1-19.

4. Jonsson LJ, Martin C. Pretreatment of lignocellulose: formation of inhibitory by-products and strategies for minimizing their effects. Bioresour Technol. 2016;199:103-12.

5. Woiciechowski AL, Neto CJD, Vandenberghe LPD, Neto DPD, Sydney ACN, Letti LA, Karp SG, Torres LAZ, Soccol CR. Lignocellulosic biomass: Acid and alkaline pretreatments and their effects on biomass recalcitrance - Conventional processing and recent advances. Bioresour Technol. 2020;304:1.

6. Kim D. Physico-chemical conversion of lignocellulose: inhibitor effects and detoxification strategies: a mini review. Molecules. 2018;23:1.

7. Liu ZL. Molecular mechanisms of yeast tolerance and in situ detoxification of lignocellulose hydrolysates. Appl Microbiol Biotechnol. 2011;90:809-25.

8. Almeida JRM, Modig T, Petersson A, Hahn-Hagerdal B, Liden G, GorwaGrauslund MF. Increased tolerance and conversion of inhibitors in lignocellulosic hydrolysates by Saccharomyces cerevisiae. J Chem Technol Biotechnol. 2007:82:340-9.

9. Banerjee N, Bhatnagar R, Viswanathan L. Inhibition of glycolysis by furfural in Saccharomyces cerevisiae. Eur J Appl Microbiol. 1981:11:226-8.

10. Pampulha ME, Loureirodias MC. Combined effect of acetic-acid, PH and ethanol on intracellular PH of fermenting yeast. Appl Microbiol Biotechnol. 1989;31:547-50. 
11. Unrean P, Gatgens J, Klein B, Noack S, Champreda V. Elucidating cellular mechanisms of Saccharomyces cerevisiae tolerant to combined lignocellulosic-derived inhibitors using high-throughput phenotyping and multiomics analyses. FEMS Yeast Res. 2018;18:1.

12. Allen SA, Clark W, McCaffery JM, Cai Z, Lanctot A, Slininger PJ, Liu ZL, Gorsich SW. Furfural induces reactive oxygen species accumulation and cellular damage in Saccharomyces cerevisiae. Biotechnol Biofuels. 2010;3:1.

13. Fletcher E, Gao K, Mercurio K, Ali M, Baetz K. Yeast chemogenomic screen identifies distinct metabolic pathways required to tolerate exposure to phenolic fermentation inhibitors ferulic acid, 4-hydroxybenzoic acid and coniferyl aldehyde. Metab Eng. 2019;52:98-109.

14. Oliva JM, Ballesteros I, Negro MJ, Manzanares P, Cabanas A, Ballesteros M. Effect of binary combinations of selected toxic compounds on growth and fermentation of Kluyveromyces marxianus. Biotechnol Prog. 2004;20:715-20.

15. Zhang B, Zhang J, Wang DM, Han RX, Ding R, Gao XL, Sun LH, Hong J. Simultaneous fermentation of glucose and xylose at elevated temperatures co-produces ethanol and xylitol through overexpression of a xylose-specific transporter in engineered Kluyveromyces marxianus. Bioresour Technol. 2016;216:227-37.

16. Kumar V, Yadav SK, Kumar J, Ahluwalia V. A critical review on current strategies and trends employed for removal of inhibitors and toxic materials generated during biomass pretreatment. Bioresour Technol. 2020;299:122633.

17. Zhang J, Zhang B, Wang D, Gao X, Hong J. Xylitol production at high temperature by engineered Kluyveromyces marxianus. Bioresour Technol. 2014;152C:192-201.

18. Zhang J, Zhang B, Wang DM, Gao XL, Hong J. Improving xylitol production at elevated temperature with engineered Kluyveromyces marxianus through over-expressing transporters. Bioresour Technol. 2015;175:642-5.

19. Zhang J, Zhang B, Wang DM, Gao XL, Sun LH, Hong J. Rapid ethanol production at elevated temperatures by engineered thermotolerant Kluyveromyces marxianus via the NADP(H)-preferring xylose reductasexylitol dehydrogenase pathway. Metab Eng. 2015;31:140-52.

20. Nonklang S, Abdel-Banat BMA, Cha-Aim K, Moonjai N, Hoshida H, Limtong S, Yamada M, Akada R. High-temperature ethanol fermentation and transformation with linear DNA in the thermotolerant Yeast Kluyveromyces marxianus DMKU3-1042. Appl Environ Microbiol. 2008;74:7514-21.

21. Banat IM, Nigam P, Marchant R. Isolation of thermotolerant, fermentative yeasts growing at 52-degrees- $C$ and producing ethanol at 45-degrees- $C$ and 50-degrees-C. World J Microbiol Biotechnol. 1992;8:259-63.

22. Groeneveld P, Stouthamer AH, Westerhoff HV. Super life - how and why "cell selection" leads to the fastest-growing eukaryote. FEBS J. 2009;276:254-70.

23. Kong X, Zhang B, Hua Y, Zhu YL, Li WJ, Wang DM, Hong J. Efficient L-lactic acid production from corncob residue using metabolically engineered thermo-tolerant yeast. Bioresour Technol. 2019;273:220-30.

24. Ballesteros I, Ballesteros M, Cabanas A, Carrasco J, Martin C, Negro MJ, Saez F, Saez R. Selection of thermotolerant yeasts for simultaneous saccharification and fermentation (SSF) of cellulose to ethanol. Appl Biochem Biotechnol. 1991;28-9:307-15.

25. Gao YS, Xu JL, Yuan ZH, Jiang JC, Zhang ZJ, Li CJ. Ethanol production from sugarcane bagasse by fed-batch simultaneous saccharification and fermentation at high solids loading. Energy Sci Eng. 2018;6:810-8.

26. Wu D, Wang D, Hong J. Effect of a novel alpha/beta hydrolase domain protein on tolerance of $K$. marxianus to lignocellulosic biomass derived inhibitors. Front Bioeng Biotechnol. 2020;8:844.

27. Wang DM, Wu D, Yang XX, Hong J. Transcriptomic analysis of thermotolerant yeast Kluyveromyces marxianus in multiple inhibitors tolerance. RSC Adv. 2018:8:14177-92.

28. Oliva JM, Saez F, Ballesteros I, Gonzalez A, Negro MJ, Manzanares P, Ballesteros M. Effect of lignocellulosic degradation compounds from steam explosion pretreatment on ethanol fermentation by thermotolerant yeast Kluyveromyces marxianus. Appl Biochem Biotechnol. 2003;105:141-53.

29. Millan-Zambrano G, Chavez S. Nuclear functions of prefoldin. Open Biol. 2014:4:1.

30. Payan-Bravo L, Penate $X$, Chavez S: Functional Contributions of Prefoldin to Gene Expression. Prefoldins: The New Chaperones 2018, 1106:1-10.

31. Wang D, Shi W, Tang Y, Liu Y, He K, Hu Y, Li J, Yang Y, Song J. Prefoldin 1 promotes EMT and lung cancer progression by suppressing cyclin A expression. Oncogene. 2017;36:885-98.
32. Kim SY, Kim JC, Kim JK, Kim HJ, Lee HM, Choi MS, Maeng PJ, Ahn JK. Hepatitis $B$ virus $X$ protein enhances NF kappa B activity through cooperating with VBP1. BMB Rep. 2008:41:158-63.

33. Iijima M, Kano Y, Nohno T, Namba M. Cloning of cDNA with possible transcription factor activity at the G(1)-S phase transition in human fibroblast cell lines. Acta Med Okayama. 1996;50:73-7.

34. Mori K, Maeda Y, Kitaura H, Taira T, Iguchi-Ariga SMM, Ariga H. MM-1, a novel c-Myc-associating protein that represses transcriptional activity of c-Myc. J Biol Chem. 1998;273:29794-800.

35. Locascio A, Blazquez MA, Alabadi D. Dynamic regulation of cortical microtubule organization through prefoldin-DELLA interaction. Curr Biol. 2013:23:804-9.

36. Millan-Zambrano G, Rodriguez-Gil A, Penate $X$, de Miguel-Jimenez L, Morillo-Huesca M, Krogan N, Chavez S. The prefoldin complex regulates chromatin dynamics during transcription elongation. PLoS Genet. 2013;9:e1003776

37. Hong J, Wang Y, Kumagai $\mathrm{H}$, Tamaki $\mathrm{H}$. Construction of thermotolerant yeast expressing thermostable cellulase genes. J Biotechnol. 2007;130:114-23.

38. Yang C, Hu SL, Zhu SL, Wang DM, Gao XL, Hong J. Characterizing yeast promoters used in Kluyveromyces marxianus. World J Microbiol Biotechnol. 2015;31:1641-6.

39. Wan W, Wang DM, Gao XL, Hong J. Expression of family 3 cellulose-binding module (CBM3) as an affinity tag for recombinant proteins in yeast. Appl Microbiol Biotechnol. 2011;91:789-98.

40. Abdel-Banat BMA, Nonklang S, Hoshida H, Akada R. Random and targeted gene integrations through the control of non-homologous end joining in the yeast Kluyveromyces marxianus. Yeast. 2010;27:29-39.

41. Gao JQ, Yuan WJ, Li YM, Bai FW, Jiang Y. Synergistic effect of thioredoxin and its reductase from Kluyveromyces marxianus on enhanced tolerance to multiple lignocellulose-derived inhibitors. Microb Cell Fact. 2017;16:1.

42. Ogur M, John RS, Nagai S. Tetrazolium overlay technique for population studies of respiration deficiency in yeast. Science. 1957;125:928-9.

43. Nolan T, Hands RE, Bustin SA. Quantification of mRNA using real-time RT-PCR. Nat Protoc. 2006;1:1559-82.

44. Amberg DC, Burke DJ, Strathern JN: Yeast vital stains: DAPI stain of nuclear and mitochondrial DNA. CSH Protoc 2006, 2006.

45. Amorim AF, Pinto D, Kuras L, Fernandes L. Absence of Gim proteins, but not GimC complex, alters stress-induced transcription. BBA-Gene Regul Mech. 2017;1860:773-81.

46. Ding MZ, Wang X, Yang Y, Yuan YJ. Metabolomic study of interactive effects of phenol, furfural, and acetic acid on Saccharomyces cerevisiae. OMICS. 2011;15:647-53.

47. Vilella F, Herrero E, Torres J, de la Torre-Ruiz MA. Pkc1 and the upstream elements of the cell integrity pathway in Saccharomyces cerevisiae, Rom2 and Mtl1, are required for cellular responses to oxidative stress. J Biol Chem. 2005;280:9149-59.

48. Ibraheem O, Ndimba BK. Molecular adaptation mechanisms employed by ethanologenic bacteria in response to lignocellulose-derived inhibitory compounds. Int J Biol Sci. 2013;9:598-612.

49. Horvath IS, Franzen CJ, Taherzadeh MJ, Niklasson C, Liden G. Effects of furfural on the respiratory metabolism of Saccharomyces cerevisiae in glucose-limited chemostats. Appl Environ Microbiol. 2003;69:4076-86.

50. Jonsson LJ, Alriksson B, Nilvebrant NO. Bioconversion of lignocellulose: inhibitors and detoxification. Biotechnol Biofuels. 2013;6:1.

51. Stein LR, Imai $S$. The dynamic regulation of NAD metabolism in mitochondria. Trends Endocrinol Metab. 2012;23:420-8.

52. Sasano Y, Watanabe D, Ukibe K, Inai T, Ohtsu I, Shimoi H, Takagi H. Overexpression of the yeast transcription activator Msn2 confers furfural resistance and increases the initial fermentation rate in ethanol production. J Biosci Bioeng. 2012;113:451-5.

53. Mousnier A, Kubat N, Massias-Simon A, Segeral E, Rain JC, Benarous R, Emiliani S, Dargemont C. von Hippel-Lindau binding protein 1-mediated degradation of integrase affects HIV-1 gene expression at a postintegration step. Proc Natl Acad Sci USA. 2007;104:13615-20.

54. Narita R, Kitaura H, Torii A, Tashiro E, Miyazawa M, Ariga H, Iguchi-Ariga SMM. Rabring7 degrades c-Myc through complex formation with MM-1. PLOS ONE. 2012;7:1.

55. Parker AL, Turner N, McCarroll JA, Kavallaris M. beta III-Tubulin alters glucose metabolism and stress response signaling to promote cell survival 
and proliferation in glucose-starved non-small cell lung cancer cells, Carcinogenesis. 2016;37:787-98.

56. Parker AL, Kavallaris M, McCarroll JA. Microtubules and their role in cellular stress in cancer. Front Oncol. 2014;4:153.

57. Miura Y, Kano M, Abe K, Urano S, Suzuki S, Toda T. Age-dependent variations of cell response to oxidative stress: proteomic approach to protein expression and phosphorylation. Electrophoresis. 2005;26:2786-96.

58. Jo WJ, Loguinov A, Wintz H, Chang M, Smith AH, Kalman D, Zhang LP, Smith MT, Vulpe CD. Comparative functional genomic analysis identifies distinct and overlapping sets of genes required for resistance to monomethylarsonous acid (MMA(III)) and arsenite (As-III) in yeast. Toxicol Sci. 2009;111:424-36.

59. Cohnen A, Bielig H, Hollenberg CP, Hu Z, Ramezani-Rad M. The Yeast ubiquitin-like domain protein Mdy2 is required for microtubule-directed nuclear migration and localizes to cytoplasmic granules in response to heat stress. Cytoskeleton. 2010;67:635-49.

60. Sato M, Kobori H, Ishijima SA, Feng ZH, Hamada K, Shimada S, Osumi M. Schizosaccharomyces pombe is more sensitive to pressure stress than Saccharomyces cerevisiae. Cell Struct Funct. 1996;21:167-74.

61. Chowdhury S, Smith KW, Gustin MC. Osmotic-stress and the yeast cytoskeleton - phenotype-specific suppression of an actin mutation. $J$ Cell Biol. 1992;118:561-71.
62. Homoto S, Izawa S. Persistent actin depolarization caused by ethanol induces the formation of multiple small cortical septin rings in yeast. $J$ Cell Sci. 2018;131:1.

63. Caspeta L, Castillo T, Nielsen J. Modifying yeast tolerance to inhibitory conditions of ethanol production processes. Front Bioeng Biotech. 2015;3:1.

64. Fu SY, Hu JF, Liu H. Inhibitory effects of biomass degradation products on ethanol fermentation and a strategy to overcome them. BioResources. 2014;9:4323-35.

65. Ando S, Arai I, Kiyoto K, Hanai S. Identification of aromatic monomers in steam-exploded poplar and their influences on ethanol fermentation by Saccharomyces-Cerevisiae. J Ferment Bioeng. 1986;64:567-70.

\section{Publisher's Note}

Springer Nature remains neutral with regard to jurisdictional claims in published maps and institutional affiliations.
Ready to submit your research? Choose BMC and benefit from:

- fast, convenient online submission

- thorough peer review by experienced researchers in your field

- rapid publication on acceptance

- support for research data, including large and complex data types

- gold Open Access which fosters wider collaboration and increased citations

- maximum visibility for your research: over $100 \mathrm{M}$ website views per year

At BMC, research is always in progress.

Learn more biomedcentral.com/submissions 